\title{
History of the solar-type protostar IRAS 16293-2422 as told by the cyanopolyynes
}

\author{
A. Jaber Al-Edhari ${ }^{1,2}$, C. Ceccarelli ${ }^{1}$, C. Kahane $^{1}$, S. Viti ${ }^{3}$, N. Balucani ${ }^{4,1}$, E. Caux ${ }^{5,6}$, A. Faure ${ }^{1}$, B. Lefloch ${ }^{1}$, \\ F. Lique ${ }^{7}$, E. Mendoza ${ }^{8}$, D. Quenard ${ }^{5,6}$, and L. Wiesenfeld ${ }^{1}$
}

1 Univ. Grenoble Alpes, CNRS, IPAG, 38000 Grenoble, France e-mail: [ali.al-edhari; cecilia.ceccarelli] @univ-grenoble-alpes.fr

2 University of AL-Muthanna, College of Science, Physics Department, 38028 AL-Muthanna, Iraq

3 University College London, Gower Street, London WC1E 6BT, UK

4 Dipartimento di Chimica, Biologia e Biotecnologie, 06123 Perugia, Italy

5 Université de Toulouse, UPS-OMP, IRAP, 31400 Toulouse, France

${ }^{6}$ CNRS, IRAP, 9 Av. Colonel Roche, BP 44346, 31028 Toulouse Cedex 4, France

7 LOMC - UMR 6294, CNRS-Université du Havre, 25 rue Philippe Lebon, BP 1123, 76063 Le Havre, France

${ }^{8}$ Instituto de Astronomia, Geofísica e Ciencias Atmosféricas, Universidade de São Paulo, 05508-090 São Paulo, SP, Brazil

Received 9 August 2016/ Accepted 22 September 2016

\begin{abstract}
Context. Cyanopolyynes are chains of carbon atoms with an atom of hydrogen and a CN group on either side. They are detected almost everywhere in the interstellar medium (ISM), as well as in comets. In the past, they have been used to constrain the age of some molecular clouds, since their abundance is predicted to be a strong function of time. Finally, cyanopolyynes can potentially contain a large portion of molecular carbon.

Aims. We present an extensive study of the cyanopolyynes distribution in the solar-type protostar IRAS 16293-2422. The goals are (i) to obtain a census of the cyanopolyynes in this source and of their isotopologues; (ii) to derive how their abundance varies across the protostar envelope; and (iii) to obtain constraints on the history of IRAS 16293-2422 by comparing the observations with the predictions of a chemical model.

Methods. We analysed the data from the IRAM-30 m unbiased millimeter and submillimeter spectral survey towards IRAS 162932422 named TIMASSS. The derived spectral line energy distribution (SLED) of each detected cyanopolyyne was compared with the predictions from the radiative transfer code GRenoble Analysis of Protostellar Envelope Spectra (GRAPES) to derive the cyanopolyyne abundances across the envelope of IRAS 16293-2422. Finally, the derived abundances were compared with the predictions of the chemical model UCL_CHEM.

Results. We detect several lines from cyanoacetylene $\left(\mathrm{HC}_{3} \mathrm{~N}\right)$ and cyanodiacetylene $\left(\mathrm{HC}_{5} \mathrm{~N}\right)$, and report the first detection of deuterated cyanoacetylene, $\mathrm{DC}_{3} \mathrm{~N}$, in a solar-type protostar. We found that the $\mathrm{HC}_{3} \mathrm{~N}$ abundance is roughly constant $\left(\sim 1.3 \times 10^{-11}\right)$ in the outer cold envelope of IRAS 16293-2422, and it increases by about a factor 100 in the inner region where the dust temperature exceeds $80 \mathrm{~K}$, namely when the volcano ice desorption is predicted to occur. The $\mathrm{HC}_{5} \mathrm{~N}$ has an abundance similar to $\mathrm{HC}_{3} \mathrm{~N}$ in the outer envelope and about a factor of ten lower in the inner region. The comparison with the chemical model predictions provides constraints on the oxygen and carbon gaseous abundance in the outer envelope and, most importantly, on the age of the source. The $\mathrm{HC}_{3} \mathrm{~N}$ abundance derived in the inner region, and where the increase occurs, also provide strong constraints on the time taken for the dust to warm up to $80 \mathrm{~K}$, which has to be shorter than $\sim 10^{3}-10^{4} \mathrm{yr}$. Finally, the cyanoacetylene deuteration is about $50 \%$ in the outer envelope and $\leq 5 \%$ in the warm inner region. The relatively low deuteration in the warm region suggests that we are witnessing a fossil of the $\mathrm{HC}_{3} \mathrm{~N}$ abundantly formed in the tenuous phase of the pre-collapse and then frozen into the grain mantles at a later phase.

Conclusions. The accurate analysis of the cyanopolyynes in IRAS 16293-2422 unveils an important part of its past story. It tells us that IRAS $16293-2422$ underwent a relatively fast $\left(\leq 10^{5} \mathrm{yr}\right)$ collapse and a very fast $\left(\leq 10^{3}-10^{4} \mathrm{yr}\right)$ warming up of the cold material to $80 \mathrm{~K}$.
\end{abstract}

Key words. astrochemistry - ISM: abundances - ISM: individual objects: IRAS 16293-2422

\section{Introduction}

Cyanopolyynes, $\mathrm{H}-\mathrm{C}_{2 n}-\mathrm{CN}$, are linear chains of $2 n$ carbons bonded at the two extremities with a hydrogen atom and a $\mathrm{CN}$ group, respectively. They seem to be ubiquitous in the interstellar medium (ISM), as they have been detected in various environments, from molecular clouds to late-type carbonrich asymptotic giant branch (AGB) stars. The detection of the largest cyanopolyyne, $\mathrm{HC}_{11} \mathrm{~N}$, has been reported and then dismissed in the molecular cloud TMC-1 (Bell et al. 1997; Loomis et al. 2016), which shows an anomalously large abundance of cyanopolyynes with respect to other molecular clouds. Curiously enough, in star-forming regions, only relatively short chains have been reported in the literature so far, up to $\mathrm{HC}_{7} \mathrm{~N}$, in dense cold cores and warm carbon-chain chemistry (WCCC) sources (e.g. Sakai et al. 2008; Cordiner et al. 2012; Friesen et al. 2013). For many years, cyanopolyynes have been suspected to be possible steps in the synthesis of simple amino 
acids (e.g. Brack 1998). More recently, the rich $N$-chemistry leading to large cyanopolyynes chains observed in Titan has renewed the interest in this family of molecules, as Titan is claimed to be a possible analogue of the early Earth (Lunine 2009). An important property of cyanopolyynes that is particularly relevant for astrobiological purposes is that they are more stable and robust against the harsh interstellar environment compared to their monomer, and hence they may better resist the exposure to UV and cosmic rays (Clarke \& Ferris 1995). Very recent observations towards the comet $67 \mathrm{P} /$ Churyumov-Gerasimenko by Rosetta seem to support the idea that cyanide polymers, and, by analogy, cyanopolyynes, are abundant on the comet's surface (Goesmann et al. 2015).

Regardless of the role of cyanopolyynes in prebiotic chemistry, when trapped in interstellar ices, they can certainly carry large quantities of carbon atoms. It is, therefore, of interest to understand in detail their formation, carbon chain accretion, and evolution in solar-like star-forming regions. The goal of this article is to provide the first census of cyanopolyynes in a solar-like protostar. To this end, we used the 3-1 mm unbiased spectral survey TIMASSS (Sect. 3) towards the well-studied protostar IRAS 16293-2422 (Sect. 2), to derive the abundance across the envelope and hot corino of $\mathrm{HC}_{3} \mathrm{~N}, \mathrm{HC}_{5} \mathrm{~N}$ and their respective isotopologues (Sect. 4). The measured abundance profiles provide us with constraints on the formation routes of these species (Sect. 5). Section 6 discusses the implications of the analysis, and Sect. 7 summarises our conclusions.

\section{Source description}

IRAS 16293-2422 (hereafter IRAS 16293) is a solar-type Class 0 protostar in the $\rho$ Ophiuchus star-forming region, at a distance of 120 pc (Loinard et al. 2008). It has a bolometric luminosity of $22 L_{\odot}$ (Crimier et al. 2010). Given its proximity and brightness, it has been the target of numerous studies that have reconstructed its physical and chemical structure. Briefly, IRAS 16293 has a large envelope that extends up to $\sim 6000$ AU and that surrounds two sources, named I16293-A and I16293-B in the literature, separated by $\sim 5^{\prime \prime}$ ( $600 \mathrm{AU}$; Wootten 1989; Mundy et al. 1992). I16293-A sizes are $\sim 1^{\prime \prime}$, whereas I16293-B is unresolved at a scale of $\sim 0.4^{\prime \prime}$ (Zapata et al. 2013). I16293-A itself is composed of at least two sources, each one emitting a molecular outflow (Mizuno et al. 1990; Loinard et al. 2013). I16293-B possesses a very compact outflow (Loinard et al. 2013) and is surrounded by infalling gas (Pineda et al. 2012; Zapata et al. 2013). From a chemical point of view, IRAS 16293 can be considered as composed of an outer envelope, characterised by low molecular abundances, and a hot corino, where the abundance of many molecules increases by orders of magnitude (e.g. Ceccarelli et al. 2000; Schöier et al. 2002; Cazaux et al. 2003; Jaber et al. 2014). The transition between the two regions is presumed to occur at $\sim 100 \mathrm{~K}$, which is the sublimation temperature of the icy grain mantles.

\section{Data set}

\subsection{Observations}

We used data from The IRAS 16293 Millimeter And Submillimeter Spectral Survey (TIMASSS; Caux et al. 2011). Briefly, the survey covers the $80-280 \mathrm{GHz}$ frequency interval and it has been obtained at the IRAM-30 m during the period January 2004 to August $2006(\sim 200 \mathrm{~h})$. Details on the data reduction and calibration can be found in Caux et al. (2011). We recall here the main features that are relevant for this work. The telescope beam depends on the frequency and varies between $9^{\prime \prime}$ and $30^{\prime \prime}$. The spectral resolution varies between 0.3 and $1.25 \mathrm{MHz}$, corresponding to velocity resolutions between 0.51 and $2.25 \mathrm{~km} \mathrm{~s}^{-1}$. The achieved rms ranges from 4 to $17 \mathrm{mK}$. We note that it is given in a $1.5 \mathrm{~km} \mathrm{~s}^{-1}$ bin for observations taken with a velocity resolution $\leq 1.5 \mathrm{~km} \mathrm{~s}^{-1}$, and in the resolution bin for higher velocity resolutions. The observations are centered on I16293-B at $\alpha(2000.0)=16^{\mathrm{h}} 32^{\mathrm{m}} 22^{\mathrm{s}} .6$ and $\delta(2000.0)=-24^{\circ} 28^{\prime} 33^{\prime \prime}$. We note that the I16293-A and I16293-B components are both inside the beam of the observations at all frequencies.

\subsection{Species identification}

We searched for the lines of cyanopolyynes and their isotopologues using the spectroscopic databases Jet Propulsion Laboratory (JPL; Pickett et al. 1998) and the Cologne Database for Molecular Spectroscopy (CDMS; Müller et al. 2005). We then used the package CASSIS (Centre d'Analyse Scientifique de Spectres Instrumentaux et Synthétiques) ${ }^{1}$ to fit a Gaussian to the lines. For the line identification we adopted the following criteria:

1. The line is detected with more than $3 \sigma$ in the integrated line intensity.

2. The line is not blended with other molecular lines.

3. The line intensity is compatible with the spectral line energy distribution (SLED): since cyanopolyyynes are linear molecules, their SLED is a smooth curve, so that lines are discarded when their intensities are out of the SLED defined by the majority of the lines, as shown in Fig. A.1.

4. The full width at half-maximum (FWHM) of the line is similar to that of the other cyanopolyyne lines with a similar upper level energy. In practice, a posteriori the FWHM is about $2 \mathrm{~km} \mathrm{~s}^{-1}$ for lines with $J \leq 12$ and increases to $6-7 \mathrm{~km} \mathrm{~s}^{-1}$ for $J=22-30$ lines.

5. The line rest velocity $V_{\mathrm{LRS}}$ does not differ by more than $0.5 \mathrm{~km} \mathrm{~s}^{-1}$ from the $V_{\mathrm{LRS}}$ of the other cyanopolyyne lines.

The first two criteria lead to the detection of 18 lines of $\mathrm{HC}_{3} \mathrm{~N}$ (from $J=9-8$ to $J=30-29$ ), 9 lines of $\mathrm{HC}_{5} \mathrm{~N}$ (from $J=31-30$ to $J=39-38$ ), and the detection of 6 lines of $\mathrm{DC}_{3} \mathrm{~N}$ (from $J=10-9$ to $J=17-16$ ). The full list of detected lines with their spectroscopic parameters is reported in Table 1. All the spectra are shown in Figs. 1-3 for $\mathrm{HC}_{3} \mathrm{~N}, \mathrm{HC}_{5} \mathrm{~N}$, and $\mathrm{DC}_{3} \mathrm{~N}$, respectively. We did not detect any line from the ${ }^{13} \mathrm{C}$ isotopologues of $\mathrm{HC}_{3} \mathrm{~N}$.

We applied the remaining three criteria and then discarded four $\mathrm{HC}_{3} \mathrm{~N}$ lines (at 109.173, 245.606, 254.699, and $263.792 \mathrm{GHz}$ ) that had calibration problems (Caux et al. 2011) and/or were blended with unidentified species (see Fig. A.1). Similarly, one $\mathrm{HC}_{5} \mathrm{~N}$ line (at $95.850 \mathrm{GHz}$ ) was discarded because of its abnormal values of the intensity, FWHM, and velocity compared to the rest of the lines (see Table 1). Finally, one $\mathrm{DC}_{3} \mathrm{~N}$ (at $135.083 \mathrm{GHz}$ ) is considered as tentatively detected.

In summary, we clearly detected $\mathrm{HC}_{3} \mathrm{~N}, \mathrm{HC}_{5} \mathrm{~N}$, and $\mathrm{DC}_{3} \mathrm{~N}$, for which 14, 8, and 6 lines were identified following the five criteria above. We did not detect any ${ }^{13} \mathrm{C}$ isotopologue of $\mathrm{HC}_{3} \mathrm{~N}$, nor cyanopolyynes larger than $\mathrm{HC}_{5} \mathrm{~N}$.

\footnotetext{
1 http://cassis.irap.omp.eu
} 
A. Jaber Al-Edhari et al.: History of the solar-type protostar IRAS 16293-2422 as told by the cyanopolyynes
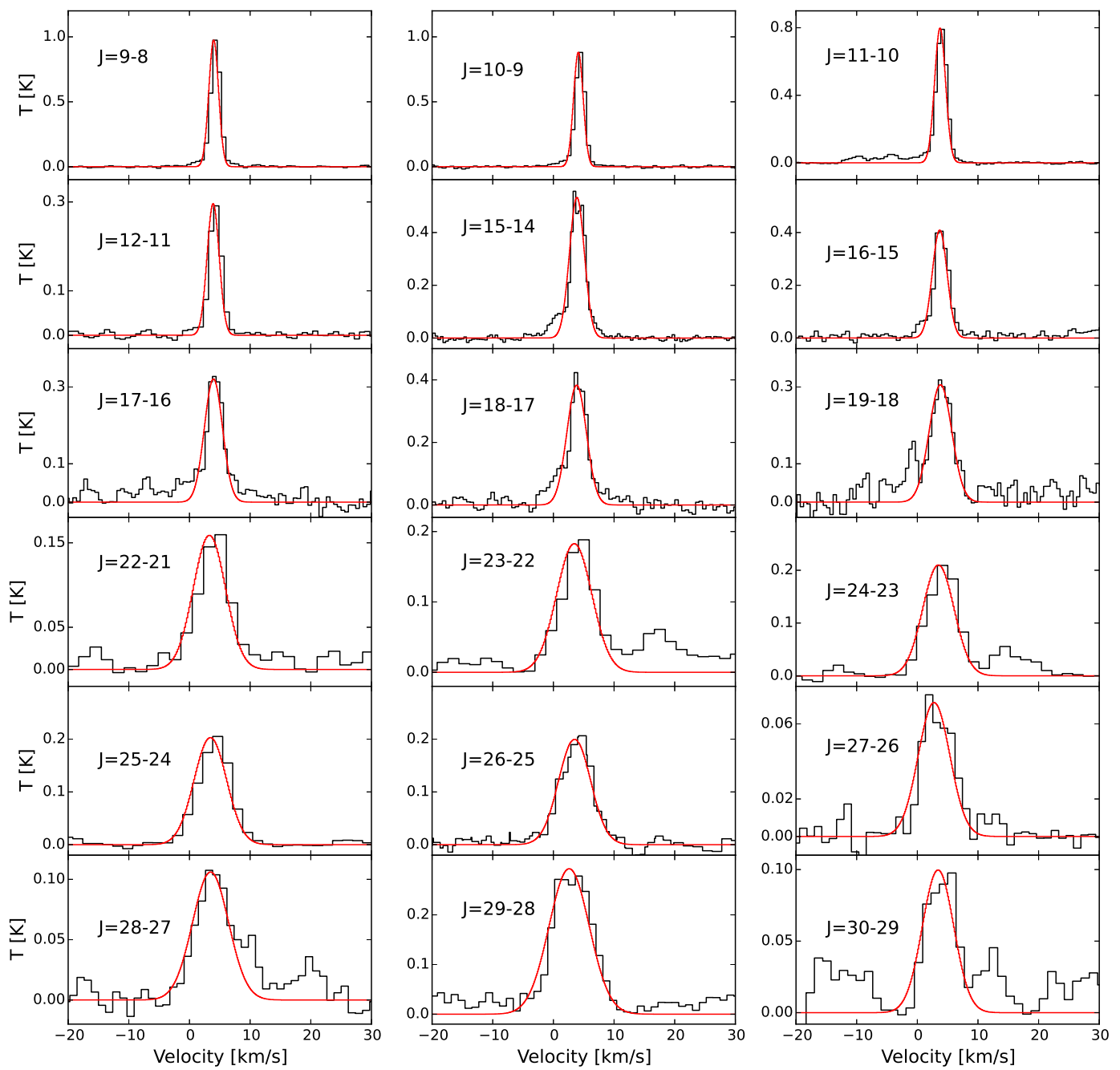

Fig. 1. Observed spectra of the detected lines of $\mathrm{HC}_{3} \mathrm{~N}$. The red curves show the Gaussian fits. The temperature is a main-beam antenna temperature.
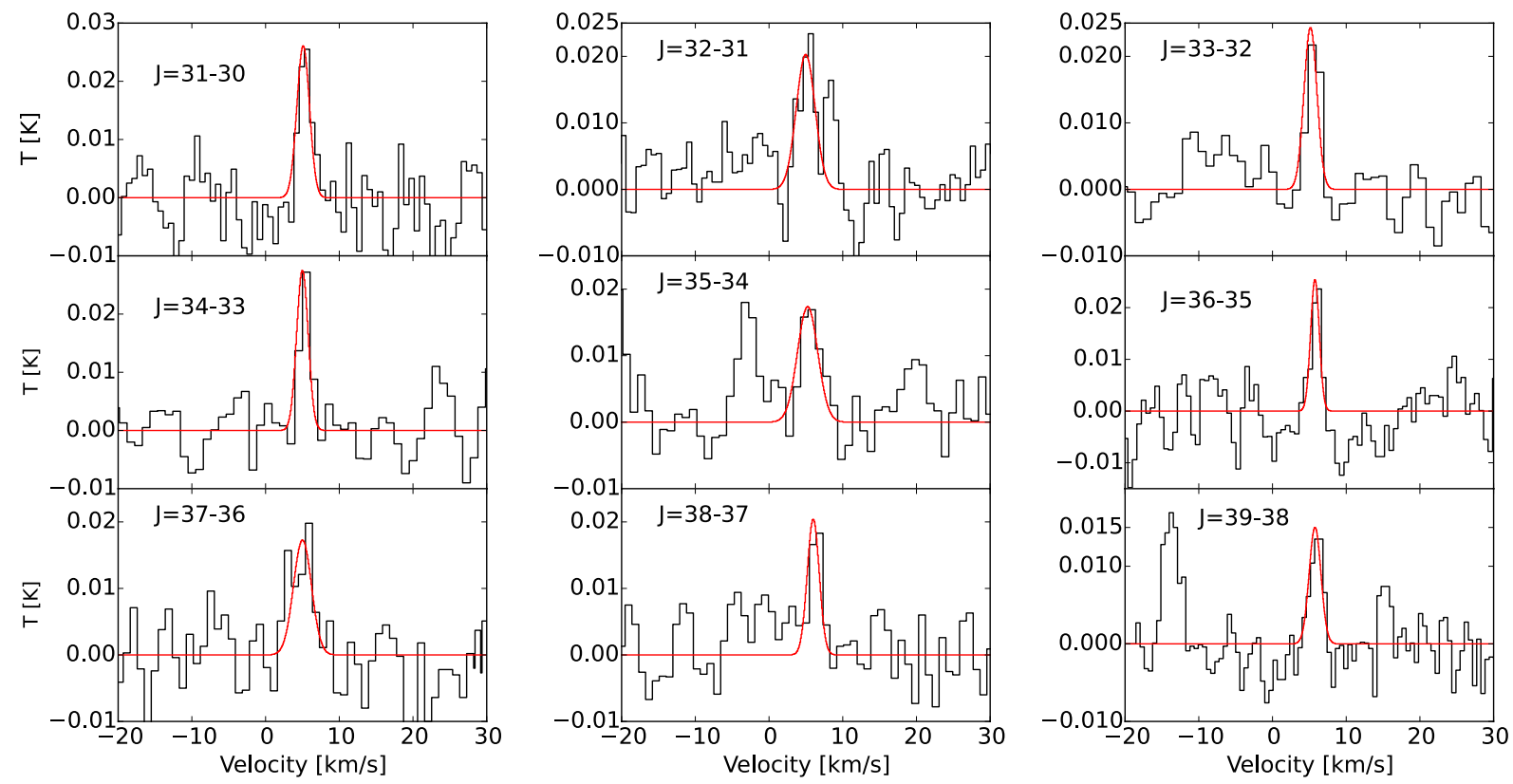

Fig. 2. Observed spectra of the detected lines of $\mathrm{HC}_{5} \mathrm{~N}$. The red curves show the Gaussian fits. The temperature is a main-beam antenna temperature. 

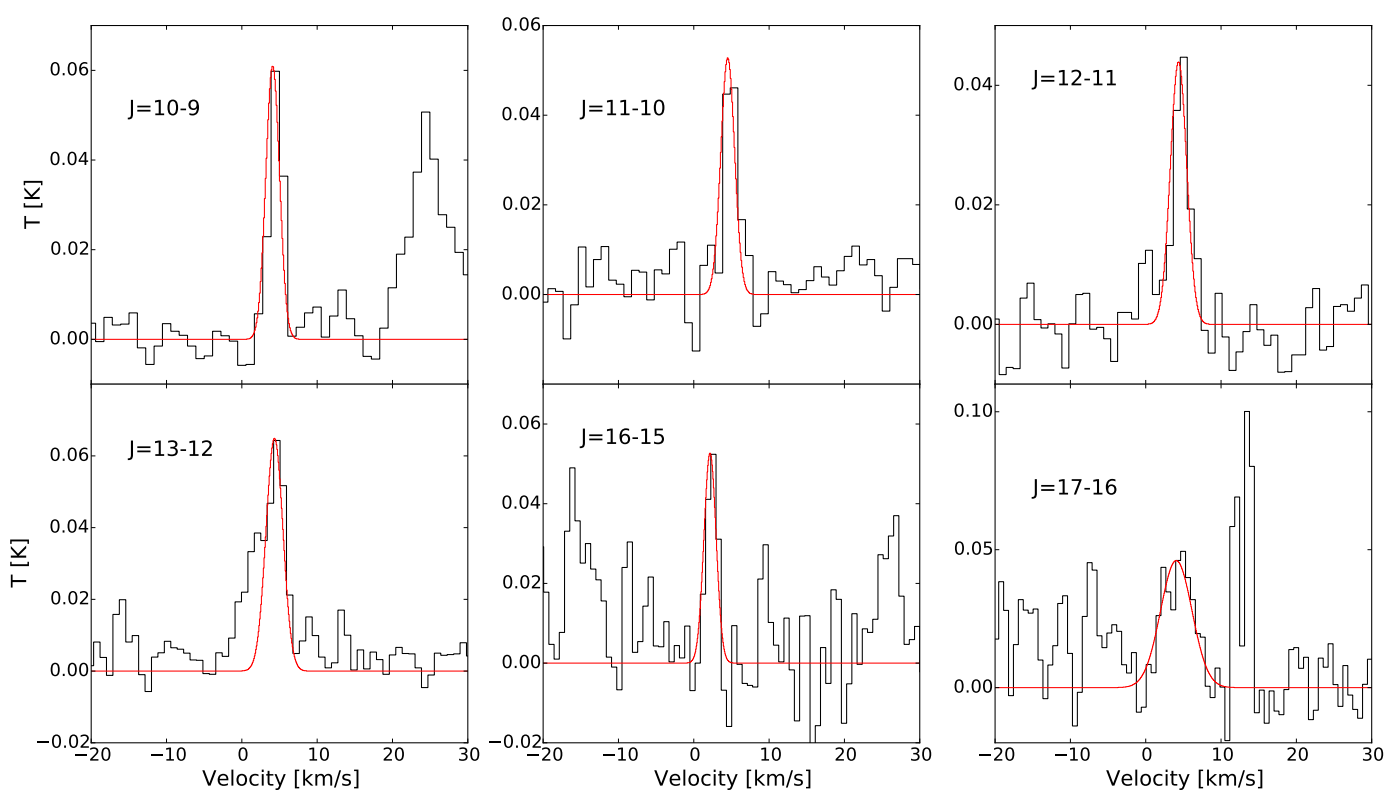

Fig. 3. Observed spectra of the detected lines of $\mathrm{DC}_{3} \mathrm{~N}$. The red curves show the Gaussian fits. The temperature is a main-beam antenna temperature.

\section{Line modelling}

\subsection{Model description}

As in our previous work (Jaber et al. 2014), we used the package GRAPES (GRenoble Analysis of Protostellar Envelope Spectra), which is based on the code described in Ceccarelli et al. (1996, 2003), to interpret the SLED of the detected cyanopolyynes. Briefly, GRAPES computes the SLED from a spherical infalling envelope for a given density and temperature structure. The code uses the beta escape probability formalism to locally solve the level population statistical equilibrium equations and consistently computes each line optical depth by integrating it over the solid angle at each point of the envelope. The predicted line flux is then integrated over the whole envelope after convolution with the telescope beam. The abundance $X$ (with respect to $\mathrm{H}_{2}$ ) of the considered species is assumed to vary as a function of the radius with a power law $\alpha$ in the cold part of the envelope, and as a jump to a new abundance in the warm part, corresponding to the sublimation of ices (e.g. Caselli \& Ceccarelli 2012). The transition between the two regions is set by the dust temperature, to simulate the sublimation of the ice mantles, and occurs at $T_{\text {jump. }}$.

The abundance is therefore given by

$$
\begin{array}{ll}
X(r)=X_{\text {out }}\left(\frac{r}{R_{\max }}\right)^{\alpha} & T_{\text {dust }} \leq T_{\text {jump }} \\
X(r)=X_{\text {in }} & T_{\text {dust }}>T_{\text {jump }},
\end{array}
$$

where $R_{\max }$ represents the largest radius of the envelope. We here used the physical structure of the IRAS 16293 envelope derived by Crimier et al. (2010), where the maximum radius is $1 \times 10^{17} \mathrm{~cm}$.

We carried out non-local thermal equilibrium (NLTE) calculations for all detected species. We used the collisional coefficients for $\mathrm{HC}_{3} \mathrm{~N}$ computed by Faure et al. (2016). For $\mathrm{DC}_{3} \mathrm{~N}$ collisional coefficients, we assumed the same as those of $\mathrm{HC}_{3} \mathrm{~N}$. Finally, the collisional coefficients for $\mathrm{HC}_{5} \mathrm{~N}$ have been computed by Lique et al. (in prep.). Briefly, $\mathrm{HC}_{5} \mathrm{~N}$ rate coefficients were extrapolated from HCN (Ben Abdallah et al. 2012) and $\mathrm{HC}_{3} \mathrm{~N}$ (Wernli et al. 2007) considering that the cyanopolyyne rate coefficients are proportional to the size of the molecules as first suggested by Snell et al. (1981). However, to improve the accuracy of the estimation, we considered scaling factors that also depend on the transition and the temperature. Hence, the ratio of $\mathrm{HC}_{3} \mathrm{~N}$ to $\mathrm{HCN}$ rate coefficients was also used to evaluate the $\mathrm{HC}_{5} \mathrm{~N}$ coefficients as follows:

$k_{H C_{5} N}(T)=k_{\mathrm{HC}_{3} \mathrm{~N}}(T) *\left[\frac{1}{2}+\frac{1}{2} \frac{k_{\mathrm{HC}_{3} \mathrm{~N}}(T)}{k_{H C N}(T)}\right]$.

We assumed the Boltzmann value for the ortho-to-para ratio of $\mathrm{H}_{2}$. Finally, we note that the disadvantage of GRAPES, namely its inability to compute the emission separately for the two sources I16293-A and I16293-B (Jaber et al. 2014), can be neglected here because previous interferometric observations by Chandler et al. (2005) and Jørgensen et al. (2011) have demonstrated that the cyanopolyyne line emission arises from source I16293-A, as also found by the analysis by Caux et al. (2011).

We ran large grids of models varying the four parameters, $X_{\text {in }}, X_{\text {out }}, \alpha$, and $T_{\text {jump }}$, and found the best fit to the observed fluxes. In general, we explored the $X_{\text {in }}-X_{\text {out }}$ parameter space by running $10 \times 10$ and $20 \times 20$ grids for $\alpha$ equal to $-1,0,+1$, and +2 , and $T_{\text {jump }}$ from 10 to $200 \mathrm{~K}$ in steps of $10 \mathrm{~K}$. We note that we first started with a range of 3 or 4 orders of magnitude in $X_{\text {in }}$ and $X_{\text {out }}$ to find a first approximate solution, and then we fine-tuned the grid around it.

\subsection{Results}

\subsection{1. $\mathrm{HC}_{3} \mathrm{~N}$}

The $\mathrm{HC}_{3} \mathrm{~N}$ SLED has been analysed in two steps, as follows.

Step 1: we first ran a grid of models varying $T_{\text {jump }}, X_{\text {in }}$, and $X_{\text {out }}$ with $\alpha=0$. The best fit for this first step was obtained with $T_{\text {jump }}=80 \mathrm{~K}, X_{\text {in }}=3.6 \times 10^{-10}$, and $X_{\text {out }}=6.0 \times 10^{-11}$. However, the fit is not very good (reduced $\left.\chi^{2}=1.7\right)$. We plotted for each line the predicted velocity-integrated flux emitted from a shell at a radius $r$ (namely $\mathrm{d} F / \mathrm{d} r * r$ ) as a function of the radius, as shown in Fig. A.2. For the three lines with the lowest $J$ (from 
Table 1. Parameters of the detected cyanopolyynes lines.

\begin{tabular}{|c|c|c|c|c|c|c|}
\hline Transition & $\begin{array}{c}\text { Frequency }^{c} \\
{[\mathrm{MHz}]}\end{array}$ & $\begin{array}{l}E_{\text {up }} \\
{[\mathrm{K}]}\end{array}$ & $\begin{array}{c}V_{\mathrm{LSR}} \\
{\left[\mathrm{km} \mathrm{s}^{-1}\right]}\end{array}$ & $\begin{array}{c}F W H M \\
{\left[\mathrm{~km} \mathrm{~s}^{-1}\right]}\end{array}$ & $\begin{array}{c}\text { Int. } \\
{\left[\mathrm{K} \mathrm{km} \mathrm{s}^{-1}\right]}\end{array}$ & $\begin{array}{c}\text { beam } \\
{["]}\end{array}$ \\
\hline \multicolumn{7}{|c|}{$\mathrm{HC}_{3} \mathrm{~N}$} \\
\hline $9-8^{a}$ & 81881.4 & 19.6 & $3.9(0.6)$ & $2.2(0.6)$ & $2.5(0.4)$ & 29.5 \\
\hline $10-9^{a}$ & 90979.0 & 24.0 & $3.9(0.5)$ & $2.1(0.5)$ & $2.3(0.4)$ & 26.5 \\
\hline $11-10^{a}$ & 100076.3 & 28.8 & $3.9(0.4)$ & $2.2(0.4)$ & $2.2(0.3)$ & 24.1 \\
\hline $12-11^{b}$ & 109173.6 & 34.1 & $3.9(0.4)$ & $2.3(0.4)$ & $0.9(0.1)$ & 22.1 \\
\hline $15-14$ & 136464.4 & 52.4 & $3.9(0.3)$ & $2.9(0.3)$ & $2.1(0.3)$ & 17.7 \\
\hline $16-15$ & 145560.9 & 59.4 & $3.6(0.2)$ & $2.8(0.3)$ & $1.7(0.3)$ & 16.6 \\
\hline $17-16$ & 154657.2 & 66.8 & $3.9(0.2)$ & $3.1(0.3)$ & $1.6(0.2)$ & 15.6 \\
\hline $18-17$ & 163753.3 & 74.7 & $3.9(0.2)$ & $3.5(0.3)$ & $2.1(0.3)$ & 14.7 \\
\hline $19-18$ & 172849.3 & 82.9 & $3.8(0.2)$ & $4.4(0.2)$ & $2.1(0.3)$ & 14.0 \\
\hline $22-21$ & 200135.3 & 110.5 & $3.2(0.5)$ & $6.1(0.7)$ & $1.6(0.2)$ & 12.1 \\
\hline $23-22$ & 209230.2 & 120.5 & $3.4(0.5)$ & $6.5(0.6)$ & $2.1(0.3)$ & 11.5 \\
\hline $24-23$ & 218324.7 & 131.0 & $3.4(0.5)$ & $6.1(0.5)$ & $2.2(0.3)$ & 11.1 \\
\hline $25-24$ & 227418.9 & 141.9 & $3.4(0.4)$ & $6.3(0.5)$ & $2.3(0.3)$ & 10.6 \\
\hline $26-25$ & 236512.7 & 153.2 & $3.4(0.4)$ & $6.2(0.5)$ & $2.3(0.3)$ & 10.2 \\
\hline $27-26^{b}$ & 245606.3 & 165.0 & $2.7(0.5)$ & $6.1(0.7)$ & $0.8(0.1)$ & 9.8 \\
\hline $28-27^{b}$ & 254699.5 & 177.2 & $3.4(0.4)$ & $7.0(0.4)$ & $1.5(0.2)$ & 9.5 \\
\hline $29-28^{b}$ & 263792.3 & 189.9 & $2.5(0.3)$ & $7.8(0.5)$ & $4.7(0.7)$ & 9.1 \\
\hline $30-29$ & 272884.7 & 203.0 & $3.4(0.6)$ & $6.6(1.0)$ & $1.4(0.2)$ & 8.8 \\
\hline \multicolumn{7}{|c|}{$\mathrm{HC}_{5} \mathrm{~N}^{c}$} \\
\hline $31-30$ & 82539.0 & 63.4 & $3.7(0.7)$ & $1.7(0.7)$ & $0.08(0.01)$ & 29.2 \\
\hline $32-31$ & 85201.3 & 67.5 & $3.9(0.6)$ & $2.6(0.7)$ & $0.07(0.01)$ & 28.3 \\
\hline $33-32$ & 87863.6 & 71.7 & $4.0(0.6)$ & $2.0(0.6)$ & $0.06(0.01)$ & 27.5 \\
\hline $34-33$ & 90525.9 & 76.0 & $3.9(0.6)$ & $1.8(0.6)$ & $0.06(0.01)$ & 26.7 \\
\hline $35-34$ & 93188.1 & 80.5 & $3.9(0.5)$ & $2.7(0.5)$ & $0.06(0.01)$ & 25.9 \\
\hline $36-35^{b}$ & 95850.3 & 85.1 & $4.5(0.6)$ & $1.4(0.7)$ & $0.04(0.01)$ & 25.2 \\
\hline $37-36$ & 98512.5 & 89.8 & $3.8(0.6)$ & $2.7(1.2)$ & $0.06(0.01)$ & 24.5 \\
\hline $38-37$ & 101174.7 & 94.7 & $4.6(0.5)$ & $1.8(0.6)$ & $0.05(0.01)$ & 23.9 \\
\hline $39-38$ & 103836.8 & 99.6 & $4.3(0.5)$ & $1.9(0.5)$ & $0.03(0.01)$ & 23.2 \\
\hline \multicolumn{7}{|c|}{$\mathrm{DC}_{3} \mathrm{~N}$} \\
\hline $10-9$ & 84429.8 & 22.3 & $3.9(0.6)$ & $2.1(0.6)$ & $0.17(0.06)$ & 28.6 \\
\hline $11-10$ & 92872.4 & 26.7 & $4.4(0.5)$ & $2.1(0.7)$ & $0.14(0.05)$ & 26.0 \\
\hline $12-11$ & 101314.8 & 31.6 & $4.3(0.4)$ & $2.6(0.5)$ & $0.15(0.05)$ & 23.8 \\
\hline $13-12$ & 109757.1 & 36.9 & $4.1(0.4)$ & $2.7(0.5)$ & $0.26(0.10)$ & 22.1 \\
\hline $16-15^{d}$ & 135083.1 & 55.1 & $2.1(0.3)$ & $1.7(0.4)$ & $0.13(0.05)$ & 17.7 \\
\hline $17-16$ & 143524.8 & 62.0 & $4.3(0.2)$ & $3.8(0.4)$ & $0.27(0.10)$ & 16.6 \\
\hline
\end{tabular}

Notes. The first three columns report the transition, frequency, and upper energy level $\left(E_{\text {up }}\right)$. The next three columns report the result of the Gaussian fitting: $V_{\text {LSR }}$ velocity, FWHM, and velocity-integrated line intensity (Int.). The last column reports the telescope beam at the frequency of the line. ${ }^{(a)}$ Discarded line because it is probably contaminated by the molecular cloud (see text, Sect. 4.2). ${ }^{(b)}$ Discarded line because it does not satisfy criteria 3 to 5 of Sect. 3.2. ${ }^{(c)}$ The $\mathrm{HC}_{3} \mathrm{~N}$ and $\mathrm{DC}_{3} \mathrm{~N}$ line frequencies are the same in the CDMS and JPL database. In contrast, the JPL $\mathrm{HC}_{5} \mathrm{~N}$ line frequencies are systematically overestimated with respect to those of CDMS, giving $V_{\mathrm{LSR}}$ shifted by $\sim 1 \mathrm{~km} \mathrm{~s}^{-1}$ with respect to the $V_{\mathrm{LSR}}$ derived for the $\mathrm{HC}_{3} \mathrm{~N}$ and $\mathrm{DC}_{3} \mathrm{~N}$ lines. We therefore used the $\mathrm{HC}_{5} \mathrm{~N}$ line frequencies from the CDMS database. ${ }^{(d)}$ We consider the detection of this line to be tentative.

$J=9$ to 11 ) the predicted shell-flux increases with the radius and abruptly stops at the maximum radius of the envelope. This means that these three lines are very likely contaminated by the molecular cloud, which could explain the poor $\chi^{2}$. In the second modelling step, we therefore excluded these lines and repeated the modelling, as described below.

Step 2: we ran a grid of models as in step 1 and found a better $\chi^{2}(0.8)$. We therefore extended the analysis by varying the $\alpha$ parameter between -1 and +2 , together with the other three parameters $T_{\text {jump }}, X_{\text {in }}$, and $X_{\text {out }}$. The results are reported in Fig. 4 and Table 2. We note that in Table 2, we also report the values of the abundance of the envelope, $X_{\mathrm{T} 20}$, at a radius equal to the angular size of the beam with the lowest frequencies, namely $29^{\prime \prime} .5$ in diameter. At this radius, which is $2.75 \times 10^{16} \mathrm{~cm}$ (around $1800 \mathrm{AU})$, the envelope dust temperature is $20 \mathrm{~K}$.

Figure 4 shows the $\chi^{2}$ obtained for each value of $\alpha$, minimised with respect to $X_{\text {in }}$ and $X_{\text {out }}$, as a function of $T_{\text {jump. A }} \chi^{2}$ lower than unity is obtained with $T_{\text {jump }}$ equal to $80 \mathrm{~K}$ and $\alpha$ equal to 0 and 1 (Table 2). Figure 5 shows the abundance profiles of $\mathrm{HC}_{3} \mathrm{~N}$ as predicted by the best-fit models. We note that the four models have the same $T_{\text {jump }}(80 \mathrm{~K})$, and the same $X_{\text {in }}\left(9 \times 10^{-10}\right)$ and $X_{\mathrm{T} 20}$ at $20 \mathrm{~K}\left(\sim 1 \times 10^{-11}\right)$, within a factor 2 . Therefore, the determination of $T_{\text {jump }}, X_{\text {in }}$, and $X_{\text {out }}$ at $20 \mathrm{~K}$ is very robust and depends very little on the assumption of the $\mathrm{HC}_{3} \mathrm{~N}$ abundance distribution. 

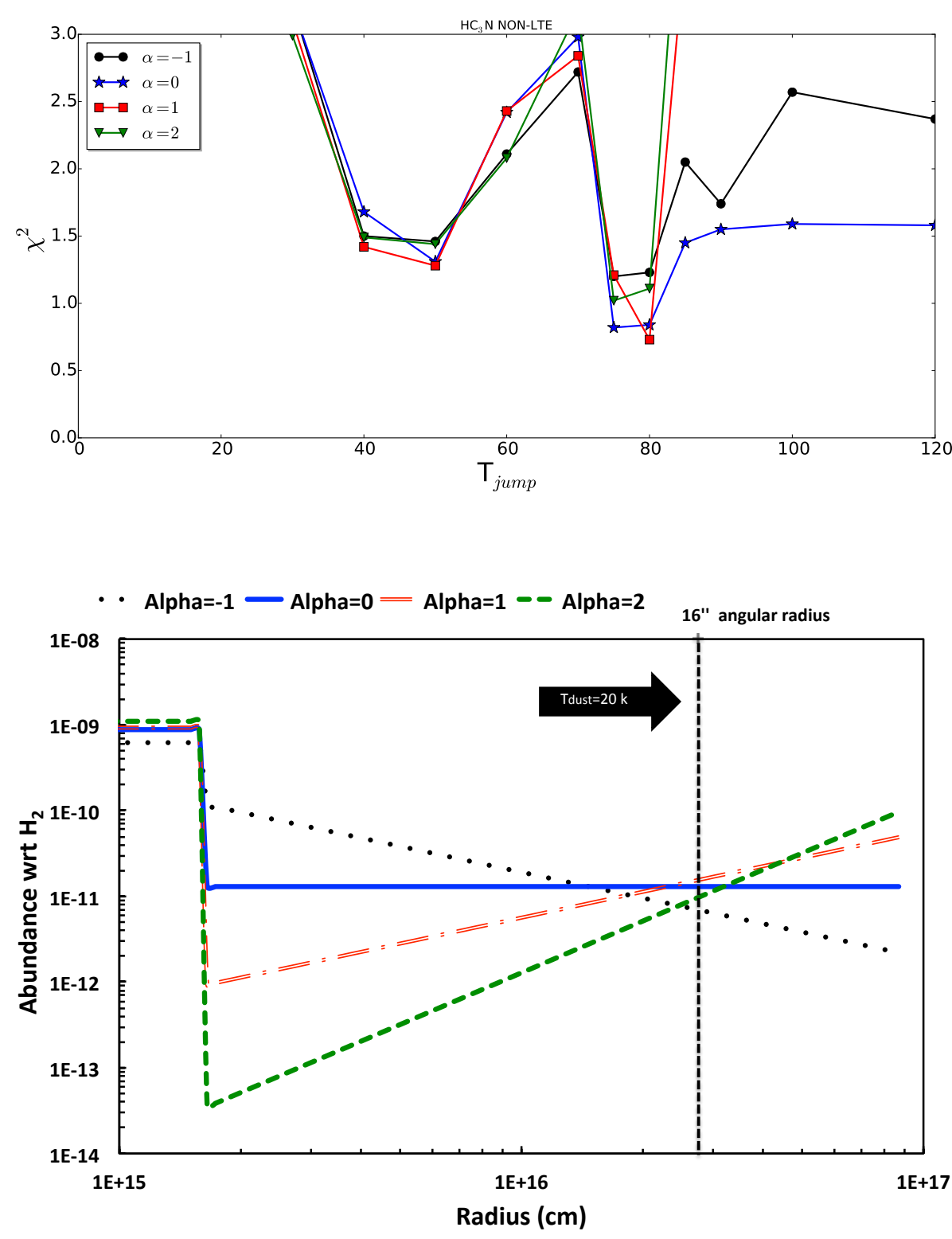

Fig. 4. Results of the $\mathrm{HC}_{3} \mathrm{~N}$ modelling. The best reduced $\chi^{2}$ optimised with respect to $X_{\text {in }}$ and $X_{\text {out }}$ as a function of $T_{\text {jump. }}$.
Fig. 5. Abundance profiles of the four $\mathrm{HC}_{3} \mathrm{~N}$ best-fit models of Table 2 .

Table 2. Results of the $\mathrm{HC}_{3} \mathrm{~N}$ modelling.

\begin{tabular}{ccccccccc}
\hline \hline Model No. & $\alpha$ & $\begin{array}{c}T_{\text {jump }} \\
{[\mathrm{K}]}\end{array}$ & $\begin{array}{c}X_{\text {in }} \\
{\left[10^{-10}\right]}\end{array}$ & $\begin{array}{c}X_{\text {out }} \\
{\left[10^{-10}\right]}\end{array}$ & $\begin{array}{c}X_{\mathrm{T} 20} \\
{\left[10^{-10}\right]}\end{array}$ & $X_{\text {in }} / X_{\text {out }}$ & $X_{\text {in }} / X_{\mathrm{T} 20}$ & $\chi^{2}$ \\
\hline Model 1 & -1 & $80 \pm 5$ & $6 \pm 1$ & $0.020_{-0.001}^{+0.009}$ & $0.07_{-0.01}^{+0.03}$ & $300_{-130}^{+70}$ & $90_{-40}^{+30}$ & 1.2 \\
Model 2 & 0 & $80 \pm 5$ & $9 \pm 1$ & $0.13_{-0.04}^{+0.03}$ & $0.13_{-0.04}^{+0.03}$ & $90_{-40}^{+20}$ & $90_{-40}^{+20}$ & 0.8 \\
Model 3 & 1 & $80 \pm 5$ & $9 \pm 1$ & $0.5_{-0.2}^{+0.1}$ & $0.2 \pm 0.1$ & $18_{-5}^{+15}$ & $45_{-20}^{+55}$ & 0.7 \\
Model 4 & 2 & $80 \pm 5$ & $11 \pm 1$ & $1.0_{-0.4}^{+0.1}$ & $0.10_{-0.04}^{+0.01}$ & $11_{-2}^{+9}$ & $110_{-20}^{+90}$ & 1.1 \\
\hline
\end{tabular}

Notes. Values of the best fit using four different values of $\alpha$.

Given the similarity of our results regardless of $\alpha$, in the following we only consider the case of $\alpha=0$. Figure A.3 shows the $\chi^{2}$ contour plots as a function of the inner $X_{\text {in }}$ and outer $X_{\text {out }}$ with $T_{\text {jump }}=80 \mathrm{~K}$. Figure A.4 reports the ratio of the observed over predicted line fluxes as a function of the upper level energy of the transition, to show the goodness of the best-fit model. Finally, Fig. A.5 shows the predicted shell-flux as a function of the radius for a sample of lines. As a final remark, we note that we also ran LTE models and obtained approximately the same results, within $20 \%$ for $X_{\text {in }}$ and $X_{\text {out }}$ and the same $T_{\text {jump }}$ and $\alpha$.

\subsection{2. $\mathrm{HC}_{5} \mathrm{~N}$}

Because we now had fewer lines, we decided to assume that $\mathrm{HC}_{5} \mathrm{~N}$ follows the spatial distribution of $\mathrm{HC}_{3} \mathrm{~N}$ and ran a grid of models with $\alpha=0$, and $T=80 \mathrm{~K}$ and $X_{\text {in }}$ and $X_{\text {out }}$ as free parameters. The $X_{\text {in }}-X_{\text {out }} \chi^{2}$ surface is shown in Fig. A.3 and the ratio between the observed and best-fit predicted intensities is shown in Fig. A.4. Table 3 summarises the best-fit values. We only obtained an upper limit to the $X_{\text {in }}$ abundance, $\leq 8 \times 10^{-11}$, while the $X_{\text {out }}$ abundance is $\sim 1 \times 10^{-11}$. When compared to $\mathrm{HC}_{3} \mathrm{~N}$, 
Table 3. Results of the analysis.

\begin{tabular}{|c|c|c|c|c|c|c|}
\hline \multirow[t]{2}{*}{ Species } & \multirow[t]{2}{*}{ Formula } & \multirow{2}{*}{$\begin{array}{c}X_{\text {in }} \\
{\left[10^{-10}\right]}\end{array}$} & \multirow{2}{*}{$\begin{array}{c}X_{\mathrm{T} 20} \\
{\left[10^{-10}\right]}\end{array}$} & \multicolumn{2}{|c|}{$X / \mathrm{HC}_{3} \mathrm{~N}$} & \multirow[t]{2}{*}{$\chi^{2}$} \\
\hline & & & & {$[\mathrm{In}]$} & {$[\mathrm{T} 20]$} & \\
\hline \multicolumn{7}{|c|}{ Detected cyanopolyynes } \\
\hline Cyanoacetylene & $\mathrm{HC}_{3} \mathrm{~N}$ & $9 \pm 1$ & $0.13_{-0.04}^{+0.03}$ & 1 & 1 & 0.8 \\
\hline Cyanodiacetylene & $\mathrm{HC}_{5} \mathrm{~N}$ & $\lesssim 0.8$ & $0.110 \pm 0.005$ & $\lesssim 0.1$ & $0.8_{-0.4}^{+0.2}$ & 1.1 \\
\hline Deuterated cyanoacetylene & $\mathrm{DC}_{3} \mathrm{~N}$ & $\lesssim 0.4$ & $0.049_{-0.003}^{+0.002}$ & $\lesssim 0.04$ & $0.4_{-0.2}^{+0.4}$ & 1.9 \\
\hline \multicolumn{7}{|c|}{ Undetected cyanopolyynes } \\
\hline Ethynylisocyanide & $\mathrm{HCCNC}$ & $\$ 7.5$ & $\lesssim 0.10$ & & & \\
\hline 3-Imino-1,2-propa-dienylidene & $\mathrm{HNCCC}$ & $\lesssim 1.2$ & $\lesssim 0.01$ & & & \\
\hline Cyanoacetylene, ${ }^{13} \mathrm{C}$ & HCCC-13-N & $\lesssim 4.5$ & $\lesssim 0.10$ & & & \\
\hline Cyanoacetylene, ${ }^{13} \mathrm{C}$ & $\mathrm{HCC}-13-\mathrm{CN}$ & $\lesssim 4.5$ & $\lessgtr 0.09$ & & & \\
\hline Cyanoacetylene, ${ }^{13} \mathrm{C}$ & $\mathrm{HC}-13-\mathrm{CCN}$ & $\lesssim 7.5$ & $\lesssim 0.15$ & & & \\
\hline \multirow[t]{2}{*}{ Cyanoacetylene, ${ }^{15} \mathrm{~N}$} & HCCCN-15 & $\lesssim 1.5$ & $\lesssim 0.15$ & & & \\
\hline & DNCCC & $\lesssim 1.5$ & $\lesssim 0.04$ & & & \\
\hline Cyanodiacetylene, ${ }^{13} \mathrm{C}$ & HCCCCC-13-N & $\lessgtr 6.0$ & $\lessgtr 0.60$ & & & \\
\hline Cyanodiacetylene, ${ }^{13} \mathrm{C}$ & HCCCC-13-CN & $\lesssim 7.5$ & $\lesssim 0.60$ & & & \\
\hline Cyanodiacetylene, ${ }^{13} \mathrm{C}$ & HCCC-13-CCN & $\lesssim 4.5$ & $\lesssim 0.45$ & & & \\
\hline Cyanodiacetylene, ${ }^{13} \mathrm{C}$ & $\mathrm{HCC}-13-\mathrm{CCCN}$ & $\lesssim 7.5$ & $\lesssim 0.75$ & & & \\
\hline Cyanodiacetylene, ${ }^{13} \mathrm{C}$ & $\mathrm{HC}-13-\mathrm{CCCCN}$ & $\lesssim 6.0$ & $\lesssim 0.60$ & & & \\
\hline Cyanodiacetylene, ${ }^{15} \mathrm{~N}$ & HCCCCCN-15 & $\lesssim 7.5$ & $\lesssim 0.75$ & & & \\
\hline Cyanodiacetylene, D & DCCCCCN & $\lesssim 7.5$ & $\lesssim 0.75$ & & & \\
\hline Cyanohexatriyne & $\mathrm{HC}_{7} \mathrm{~N}$ & $\lesssim 15$ & $\lesssim 15$ & & & \\
\hline
\end{tabular}

Notes. The first two columns report the species name and formula. Third and fourth columns report the values of the inner abundance $X_{\text {in }}$ and the abundances at $T_{\text {dust }}=20 \mathrm{~K} X_{\mathrm{T} 20}$. Columns 5 and 6 report the values of the abundance ratios $\mathrm{DC}_{3} \mathrm{~N} / \mathrm{HC}_{3} \mathrm{~N}$ and $\mathrm{HC}_{5} \mathrm{~N} / \mathrm{HC}_{3} \mathrm{~N}$ in the inner region and where $T_{\text {dust }}=20 \mathrm{~K}$, respectively. The last column reports the $\chi^{2}$ for the best fit of $\mathrm{HC}_{5} \mathrm{~N}$ and $\mathrm{DC}_{3} \mathrm{~N}$ (note that in this last case the $\chi^{2}$ is not reduced). The top half table lists the detected species, the bottom half table the upper limits to the abundance of undetected cyanopolyynes (see text, Sect. 4).

the $\mathrm{HC}_{5} \mathrm{~N}$ abundance is therefore more than ten times lower in the hot corino region, while it is the same in the outer cold envelope.

\subsection{3. $\mathrm{DC}_{3} \mathrm{~N}$}

Following the discussion on the $\mathrm{HC}_{3} \mathrm{~N}$ line analysis, we did not consider the three $\mathrm{DC}_{3} \mathrm{~N}$ lines with the lowest upper level energy, as they are likely contaminated by the molecular cloud.

For the remaining three lines, we adopted the same strategy as for $\mathrm{HC}_{5} \mathrm{~N}$ for the SLED analysis, namely we adopted $\alpha=0$, and $T=80 \mathrm{~K}$ and varied $X_{\text {in }}$ and $X_{\text {out }}$. The results are shown in Figs. A.3 and A.4, and summarised in Table 3. We obtained an upper limit of $X_{\text {in }} \leq 4 \times 10^{-11}$, while $X_{\text {out }}$ is $\leq 5 \times 10^{-12}$. This implies a deuteration ratio of $\leq 5 \%$ and $50 \%$ in the hot corino and cold envelope, respectively.

\subsubsection{Undetected species and final remarks on the observations}

As mentioned earlier, we did not detect larger cyanopolyynes or ${ }^{13} \mathrm{C}$ isotopologues. For the undetected species we derived the upper limits to the abundance by assuming that the nondetected emission arises in the hot corino (with a temperature of $80 \mathrm{~K}$, diameter $=2^{\prime \prime}, N\left(\mathrm{H}_{2}\right)=1.5 \times 10^{23} \mathrm{~cm}^{-2}$, and line $F W H M=6 \mathrm{~km} \mathrm{~s}^{-1}$ ), and cold envelope (with a temperature of $20 \mathrm{~K}$, a diameter $=30^{\prime \prime}, N\left(\mathrm{H}_{2}\right)=3.5 \times 10^{22} \mathrm{~cm}^{-2}$, and line $F W H M=3 \mathrm{~km} \mathrm{~s}^{-1}$ ), respectively. The results are listed in Table 3.

From the $\mathrm{HC}_{5} \mathrm{~N}$ and $\mathrm{DC}_{3} \mathrm{~N}$ modelling, we conclude that the detected lines of both species are dominated by the emission from the cold outer envelope, as we only obtain upper limits to their abundance in the warm inner region. This conclusion is coherent with the line widths reported in Table 1 . The $\mathrm{HC}_{3} \mathrm{~N}$ lines (see also Fig. A.1) show a clear separation between the low-energy transitions, for which the $F W H M$ is around $3 \mathrm{~km} \mathrm{~s}^{-1}$, and the high-energy transitions, for which the FWHM is around $6 \mathrm{~km} \mathrm{~s}^{-1}$ or higher. This difference strongly suggests that the two sets of lines probe different regions. As the low-energy lines correspond to $E_{\mathrm{up}}$ values lower than $80 \mathrm{~K}$, a very natural explanation is that they are dominated by emission from the cold envelope, whereas the high-energy lines $\left(E_{\text {up }} \geq 110 \mathrm{~K}\right)$ are dominated by emission from the inner warm region. As the $\mathrm{HC}_{5} \mathrm{~N}$ and $\mathrm{DC}_{3} \mathrm{~N}$ lines have a FWHM of about $3 \mathrm{~km} \mathrm{~s}^{-1}$, it is not surprising that our modelling concludes that they are dominated by emission from the cold envelope.

\section{Chemical origin of $\mathrm{HC}_{3} \mathrm{~N}$}

As discussed in the Introduction, $\mathrm{HC}_{3} \mathrm{~N}$ is an ubiquitous molecule in the ISM. There are several routes by which a high $\mathrm{HC}_{3} \mathrm{~N}$ abundance may arise. Briefly, $\mathrm{HC}_{3} \mathrm{~N}$ can form through the following neutral-neutral reactions (e.g. Wakelam et al. 2015):

$\mathrm{C}_{2} \mathrm{H}_{2}+\mathrm{CN} \longrightarrow \mathrm{HC}_{3} \mathrm{~N}+\mathrm{H}$

$\mathrm{C}_{4} \mathrm{H}+\mathrm{N} \longrightarrow \mathrm{HC}_{3} \mathrm{~N}+\mathrm{C}$

$\mathrm{C}_{3} \mathrm{H}_{2}+\mathrm{N} \longrightarrow \mathrm{HC}_{3} \mathrm{~N}+\mathrm{H}$

$\mathrm{C}_{2} \mathrm{H}+\mathrm{HNC} \longrightarrow \mathrm{HC}_{3} \mathrm{~N}+\mathrm{H}$

under typical dense-cloud conditions. Roughly, the first reaction contributes to about $80-90 \%$ of the formation of $\mathrm{HC}_{3} \mathrm{~N}$, with the other three swapping in importance depending on the initial conditions, but together contributing the remaining $20 \%$. Its 
main destruction channels are either through reactions with $\mathrm{He}^{+}$ or reactions with atomic carbon, both contributing 30-40\% to the destruction, depending on the initial conditions assumed. In dark clouds $\left(n_{\mathrm{H}} \sim 10^{4}-10^{5} \mathrm{~cm}^{-3}\right)$, it is well known that $\mathrm{HC}_{3} \mathrm{~N}$ is abundant, together with other carbon chain molecules (e.g. Suzuki et al. 1992; Caselli et al. 1998). Thus, based on its routes of formation and destruction, we would expect the $\mathrm{HC}_{3} \mathrm{~N}$ abundance to increase as a function of gas density, at least before freeze-out onto the dust grains takes over. Interestingly, however, the $\mathrm{HC}_{3} \mathrm{~N}$ abundance with respect to $\mathrm{H}_{2}$ estimated in both the envelope and in the hot corino of IRAS 16293 is rather low $\left(\sim 10^{-11}\right.$ and $\sim 10^{-9}$, respectively). In this section we therefore discuss the possible physical conditions that may lead to such low abundances of $\mathrm{HC}_{3} \mathrm{~N}$.

\subsection{Cold envelope}

First of all, we investigated qualitatively which conditions in the cold envelope may lead to a fractional abundance of $\mathrm{HC}_{3} \mathrm{~N}$ as low as $10^{-11}$. We would like to emphasise that this is difficult because very small changes in the main atomic elements $(\mathrm{O}, \mathrm{C}$, $\mathrm{N}$ ) or molecular species (CO) can lead to very large changes in the abundances of tracers species. Hence, it is likely that small uncertainties in the initial elemental abundances of the cloud as well as its depletion history (which is dependent on the time the gas remains at a particular gas density) can easily lead to differences in the abundances of species such as $\mathrm{HC}_{3} \mathrm{~N}$ of orders of magnitude.

For our analysis, we made use of the time-dependent chemical model UCL_CHEM (Viti et al. 2004). The code was used in its simplest form, namely we only included gas-phase chemistry with no dynamics (the gas was always kept at a constant density and temperature). The gas-phase chemical network is based on the UMIST database (McElroy et al. 2013) augmented with updates from the KIDA database as well as new rate coefficients for the $\mathrm{HC}_{3} \mathrm{~N}$ and $\mathrm{HC}_{5} \mathrm{~N}$ network as estimated by Loison et al. (2014). The chemical evolution was followed until chemical equilibrium was reached. To understand the chemical origin of the $\mathrm{HC}_{3} \mathrm{~N}$ abundance that we measured in the cold envelope of IRAS $16293\left(\sim 10^{-11}\right)$, we modelled a gas density of $2 \times 10^{6} \mathrm{~cm}^{-3}$ and a gas temperature of $20 \mathrm{~K}$, which are the values inferred by Crimier et al. (2010) at a radius equivalent to the observations telescope beam (see Sect. 4.2.1).

We ran a small grid of models where we varied

- the carbon and oxygen elemental abundances in the range of $0.3-1$ and $1-3 \times 10^{-4}$, respectively;

- the nitrogen elemental abundance: 6 (solar) and $2 \times 10^{-5}$;

- the cosmic-ray ionisation rate: $5 \times 10^{-17} \mathrm{~s}^{-1}$ (assumed as our standard value) and 10 times higher.

We note that varying the $\mathrm{C}, \mathrm{O}$, and $\mathrm{N}$ elemental abundances can be considered approximately as mimicking qualitatively the degree of depletion of these elements onto the grain mantles. Furthermore, we varied the cosmic-ray ionisation rate because it is a rather uncertain parameter that could be enhanced with respect to the standard value because of the presence of X-rays or an energetic $\geq \mathrm{MeV}$ particles source embedded inside the envelope (e.g. Doty et al. 2004; Ceccarelli et al. 2014b).

The results of the modelling are plotted in Fig. 6. The top left panel is our reference model, with a standard cosmic-ray ionisation rate $\left(5 \times 10^{-17} \mathrm{~s}^{-1}\right)$, at chemical equilibrium $\left(\geq 10^{6} \mathrm{yr}\right)$, and with a solar abundance of nitrogen $\left(6.2 \times 10^{-5}\right)$. The values of the $\mathrm{HC}_{3} \mathrm{~N}$ abundances are $\log$ of the fractional abundance with respect to the total number of hydrogen nuclei, hence the

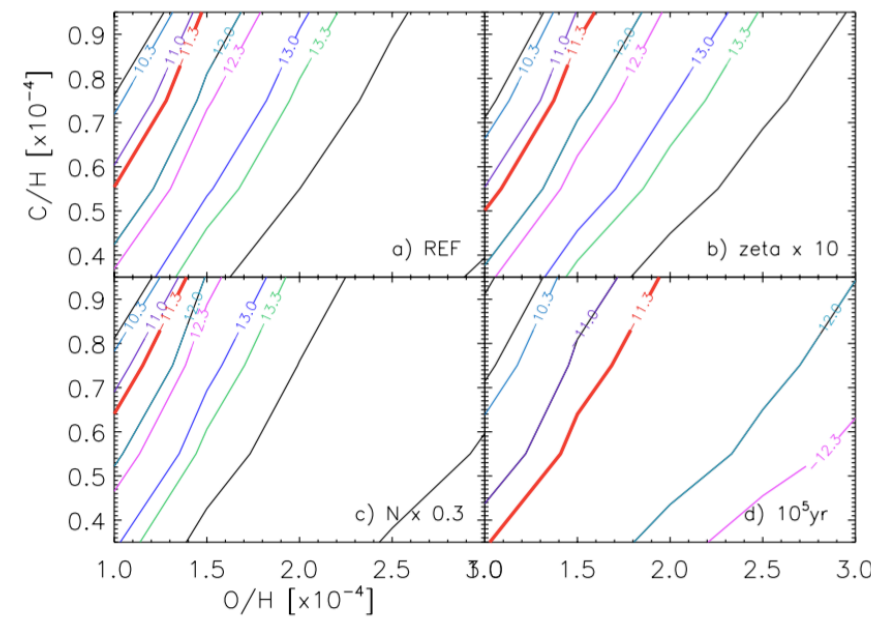

Fig. 6. Predicted $\mathrm{HC}_{3} \mathrm{~N}$ abundance (in $\log$ ) as a function of the $\mathrm{O} / \mathrm{H}$ ( $x$-axis) and $\mathrm{C} / \mathrm{H}$ ( $y$-axis) for four cases: the reference model, described in the text (upper left panel), and then the same, but with a cosmic-ray ionisation rate increased by a factor ten (upper right panel), a nitrogen elemental abundance decreased by a factor three (lower left panel) and at a time of $10^{5} \mathrm{yr}$ (lower right panel). The thick red lines mark the $\mathrm{HC}_{3} \mathrm{~N}$ abundance measured in the cold envelope of IRAS 16293.

best match with the observations is for a value of -11.3 . We find that this value is reached within our reference model for low values of $\mathrm{O} / \mathrm{H}\left(\leq 1.5 \times 10^{-4}\right)$ and for a $\mathrm{O} / \mathrm{C}$ ratio between 1.5 and 2 , with the lowest $\mathrm{O} / \mathrm{C}$ ratio needed with the highest $\mathrm{O} / \mathrm{H}$ abundance. This implies that both oxygen and carbon are mostly frozen onto the grain icy mantles by about the same factor, as the $\mathrm{O} / \mathrm{C}$ ratio is similar to the one in the Sun (1.8; see e.g. Asplund et al. 2009) and in the HII regions (1.4; see e.g. García-Rojas \& Esteban 2007). Increasing the cosmic-ray ionisation rate by a factor of ten changes the above conclusions little, having as an effect a slightly larger parameter space in $\mathrm{O} / \mathrm{H}$ and $\mathrm{O} / \mathrm{C}$ to reproduce the observed $\mathrm{HC}_{3} \mathrm{~N}$ abundance. In contrast, decreasing $\mathrm{N} / \mathrm{H}$ by a factor of three slightly diminishes the $\mathrm{O} / \mathrm{H}$ and $\mathrm{O} / \mathrm{C}$ parameter space. Finally, the largest $\mathrm{O} / \mathrm{H}$ and $\mathrm{O} / \mathrm{C}$ parameter space that reproduces the observed $\mathrm{HC}_{3} \mathrm{~N}$ abundance in the cold envelope of IRAS 16293 is obtained by considering earlier times, $10^{5} \mathrm{yr}$ (bottom right of Fig. 6). This possibly implies that the gas in the envelope is at a similar age to that of the protostar.

\subsection{Hot corino}

As we move towards the centre of the protostar(s), a jump in the $\mathrm{HC}_{3} \mathrm{~N}$ abundance by roughly two orders of magnitude is observed at around $80 \mathrm{~K}$. This means that the first question to answer is what this $80 \mathrm{~K}$ represents. According to the temperature programmed desorption (TPD) experiments by Collings et al. (2004) and successive similar works, in general, the ice sublimation is a complex process and does not occur at one single temperature, but in several steps. In the particular case of iced $\mathrm{HC}_{3} \mathrm{~N}$, it is expected to have two sublimation peaks, at a dust temperature of about 80 and $100 \mathrm{~K}$, respectively (for details, see Viti et al. 2004). The first corresponds to the so-called volcano ice desorption, and the second corresponds to the ice codesorption, namely the whole ice sublimation. Therefore, our measured jump temperature of $80 \mathrm{~K}$ indicates that the most important sublimation is due to the volcano desorption, while the co-desorption injects back only a minor fraction of the frozen $\mathrm{HC}_{3} \mathrm{~N}$. In addition, the $80 \mathrm{~K}$ jump also tells us that any other processes contributing to the formation of $\mathrm{HC}_{3} \mathrm{~N}$ at lower $(\leq 80 \mathrm{~K})$ 
temperatures have to be negligible. In other words, considering the reactions in the synthesis of $\mathrm{HC}_{3} \mathrm{~N}$ reported at the beginning of Sect. 5, the desorption of C-bearing iced species, for example $\mathrm{CH}_{4}$, has to provide a negligible contribution to the $\mathrm{HC}_{3} \mathrm{~N}$ abundance.

To understand what all this implies, we ran UCL_CHEM again, but this time we included freeze-out of the gas species in the cold phase to simulate the cold and dense prestellar phase. We then allowed thermal evaporation of the icy mantles as a function of the species and temperature of the dust following the recipe of Collings et al. (2004), in which the ice sublimation occurs in several steps (for details, see Viti et al. 2004). We find that the jump in the $\mathrm{HC}_{3} \mathrm{~N}$ abundance from $10^{-11}$ to $10^{-9}$ occurs only if thermal evaporation due to the volcano peak occurs quickly, on a timescale of $\leq 10^{3-4} \mathrm{yr}$, implying that the increase in the dust temperature to $\sim 80 \mathrm{~K}$ must also occur quickly, on a similar timescale. In addition, if frozen species containing $\mathrm{C}$, such as $\mathrm{CO}$ and $\mathrm{CH}_{4}$, sublimate at earlier times, namely at colder temperatures, and remain in the gas for too long before the volcano explosion, then the $\mathrm{HC}_{3} \mathrm{~N}$ abundance reaches much too high abundances too quickly. In other words, the sublimation of these species must have occurred not much before the volcano sublimation, again on timescales of $\leq 10^{3} \mathrm{yr}$.

\section{3. $\mathrm{HC}_{5} \mathrm{~N}$}

We finally note that none of our models can reproduce an $\mathrm{HC}_{5} \mathrm{~N}$ abundance comparable to the one of $\mathrm{HC}_{3} \mathrm{~N}$, as observed in the cold envelope of IRAS 16293. All models predict a much lower $\mathrm{HC}_{5} \mathrm{~N}$ abundance, by more than a factor ten, than the $\mathrm{HC}_{3} \mathrm{~N}$ abundance. Since in the warm $(T \geq 80 \mathrm{~K})$ region we only derive an upper limit for the $\mathrm{HC}_{5} \mathrm{~N}$ abundance (which is at least ten times lower than that of $\mathrm{HC}_{3} \mathrm{~N}$ ), the models cannot be constrained. The failure of our models to reproduce $\mathrm{HC}_{5} \mathrm{~N}$ in the cold envelope is most likely due to a lack of a comprehensive network for the formation and destruction of the species $\mathrm{HC}_{5} \mathrm{~N}$ and therefore calls for a revision of its gaseous chemistry. Here we briefly summarize the main routes of formation and destruction for this species in our models:

$\mathrm{C}_{2} \mathrm{H}_{2}+\mathrm{C}_{3} \mathrm{~N} \longrightarrow \mathrm{HC}_{5} \mathrm{~N}+\mathrm{H}$

$\mathrm{C}_{4} \mathrm{H}+\mathrm{HNC} \longrightarrow \mathrm{HC}_{5} \mathrm{~N}+\mathrm{H}$

contributing to its formation by $\sim 70 \%$ and $30 \%$, respectively, and

$\mathrm{H}_{3}^{+}+\mathrm{HC}_{5} \mathrm{~N} \longrightarrow \mathrm{HC}_{5} \mathrm{NH}^{+}+\mathrm{H}_{2}$

$\mathrm{HCO}^{+}+\mathrm{HC}_{5} \mathrm{~N} \longrightarrow \mathrm{HC}_{5} \mathrm{NH}^{+}+\mathrm{CO}$

contributing to its destruction by $\sim 50 \%$ and $40 \%$, respectively.

It is clear that since both $\mathrm{HC}_{3} \mathrm{~N}$ and $\mathrm{HC}_{5} \mathrm{~N}$ are tracer species (with abundances $\leq 10^{-9}$ ), at least in this particular source, a more complete network is required, possibly including cyanopolynes with a higher number of carbon than included here, together with a proper treatment of the gas-grain chemistry that is needed to properly model the cold envelope. This will be the scope of future work.

\section{Discussion}

\subsection{General remarks on cyanopolyynes in different environments}

In the Introduction, we mentioned that cyanopolyynes are almost ubiquitous in the ISM. Here we compare the cyanopolyyne abundance derived in this work with that found in various

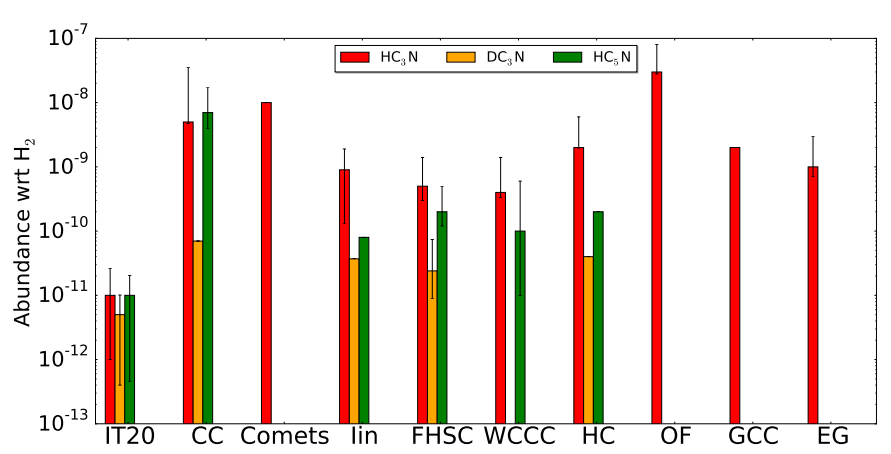

Fig. 7. Abundances of cyanopolynes in different sources: IRAS 16293 outer envelope (IT20) and inner region (Iin; this work), cold clouds (CC; Winstanley \& Nejad 1996; Miettinen 2014), comet Hale-Bopp at $1 \mathrm{AU}$ assuming $\mathrm{H}_{2} \mathrm{O} / \mathrm{H}_{2}=5 \times 10^{-5}$ (Comets; Bockelé- Morvan et al. 2000), first hydrostatic core sources (FHSC; Cordiner et al. 2012), warm carbon-chain chemistry sources (WCCC; Sakai et al. 2008, 2004), massive hot cores (HC; Schöier et al. 2002; Esplugues et al. 2013), outflow sources (OF; Bachiller \& Pérez Gutiérrez 1997; Schöier et al. 2002, Galactic Centre clouds (GCC; Marr et al. 1993; Aladro et al. 2011), and external galaxies (EG; Aladro et al. 2011).

Galactic and extragalactic environments that possess different conditions (temperature, density, and history). Figure 7 graphically shows this comparison. From this figure, we note the following: (i) $\mathrm{HC}_{3} \mathrm{~N}$ is present everywhere in the ISM with relatively high abundances; (ii) the abundance in the cold envelope of IRAS 16293 is the lowest in the plot, implying a high degree of freezing of oxygen and carbon, as indeed suggested by the chemical model analysis; (iii) $\mathrm{HC}_{3} \mathrm{~N}$ and $\mathrm{HC}_{5} \mathrm{~N}$ have relatively similar abundances in the IRAS 16293 outer envelope, cold cloud, first hydrostatic core (FHSC), and WCCC sources, namely in the cold objects of the figure, implying that the derived ratio $\mathrm{HC}_{5} \mathrm{~N} / \mathrm{HC}_{3} \mathrm{~N} \sim 1$ in the IRAS 16293 cold envelope is not, after all, a peculiarity, but probably due to the cold temperature; and finally; (iv) $\mathrm{DC}_{3} \mathrm{~N}$ is only detected in cold objects except in the warm envelope of IRAS 16293 and the hot cores, suggesting that in these sources, it is linked to the mantle sublimation, in one way or another, as again found by our chemical modelling.

\subsection{Present and past history of IRAS 16293}

Our analysis of the TIMASSS spectral survey reveals the presence of $\mathrm{HC}_{3} \mathrm{~N}, \mathrm{HC}_{5} \mathrm{~N}$, and $\mathrm{DC}_{3} \mathrm{~N}$ in IRAS $16293 . \mathrm{HC}_{3} \mathrm{~N}$ was previously detected by van Dishoeck et al. (1993), and its abundance has been analysed by Schöier et al. (2002) with a twostep abundance model, where the jump was assumed to occur at $90 \mathrm{~K}$. Schöier et al. (2002) found $\mathrm{HC}_{3} \mathrm{~N} / \mathrm{H}_{2} \sim 10^{-9}$ in the inner warm part and $\leq \sim 10^{-10}$ in the outer envelope. We note that they were unable to derive a value for the outer envelope or estimate where the jump occurs because they only detected three lines. Nonetheless, their estimates are in excellent agreement with our new estimates (Table 3).

A very important point is that we were not only able to estimate the cold envelope abundance, but also to determine where the jump occurs, an essential information for the determination of the origin of $\mathrm{HC}_{3} \mathrm{~N}$ in IRAS 16293. Our chemical modeling (Sect. 5) showed that in the cold envelope, the $\mathrm{HC}_{3} \mathrm{~N}$ abundance is reproduced for low values of oxygen and carbon in the gas phase, implying that these species are heavily frozen onto the grain mantles. The $\mathrm{O} / \mathrm{C}$ ratio is between 1.5 and 2 , similar to 


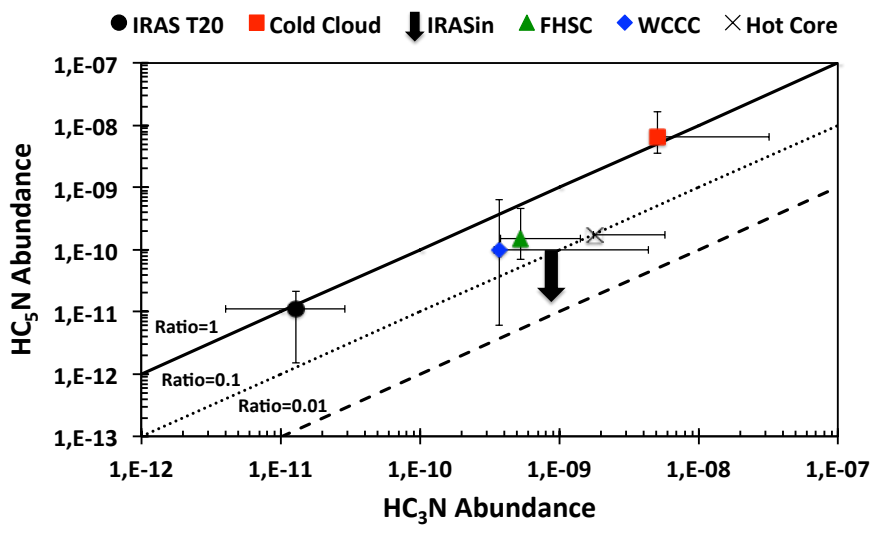

Fig. 8. Abundance of $\mathrm{HC}_{5} \mathrm{~N}$ as a function of the abundance of $\mathrm{HC}_{3} \mathrm{~N}$ in different protostellar and cold sources: inner (black arrow) and outer envelope $\left(T_{20}\right)$ of IRAS 16293 (black filled circle) presented in this work, warm carbon-chain chemistry (WCCC) sources (blue diamond; Sakai et al. 2008; Jørgensen et al. 2004), first hydrostatic core (FHSC) source (green triangle; Cordiner et al. 2012), hot cores sources (cross; Schöier et al. 2002; Esplugues et al. 2013), and Galactic Centre clouds (red square; Marr et al. 1993; Aladro et al. 2011).

solar, if the chemistry of the envelope is a fossil, namely built up during the $\sim 10^{7} \mathrm{yr}$ life of the parental molecular cloud. If, in contrast, the chemistry was reset (for any reason) and evolved in a shorter time, for instance in $10^{5} \mathrm{yr}$, the $\mathrm{O} / \mathrm{C}$ ratio that reproduces the observations could be as high as 3 , but the oxygen and carbon have anyway remained mostly frozen onto the grain mantles. Probably the most important point to remark is that the $\mathrm{HC}_{3} \mathrm{~N}$ abundance is very low, a very tiny fraction of the $\mathrm{CO}$ abundance, the main reservoir of the carbon, so that little variation in the $\mathrm{CO}$ abundance results in large variation in the $\mathrm{HC}_{3} \mathrm{~N}$ abundance.

A second extremely interesting point is that the $\mathrm{HC}_{3} \mathrm{~N}$ abundance undergoes a jump of about one hundred when the dust temperature reaches $80 \mathrm{~K}$. These two values provide us with very strong constraints on how the collapse of IRAS 16293 occurred: it must have occurred so fast that the sublimation of C-bearing ices, such as $\mathrm{CO}$ and methane, has not yet produced $\mathrm{HC}_{3} \mathrm{~N}$ in enough large abundances to mask the jump that is due to the volcano sublimation of the ices at $80 \mathrm{~K}$ (Collings et al. 2004; Viti et al. 2004). An approximate value of this time is $\sim 10^{3} \mathrm{yr}$, based on our modelling. In other words, the $\mathrm{HC}_{3} \mathrm{~N}$ measured abundance jump temperature and abundance in the warm envelope both suggest that IRAS 16293 is a very young object, as expected, and that the envelope dust heating took no more than $\sim 10^{3}$ yr to occur.

A third result from the present study is the large measured abundance of $\mathrm{HC}_{5} \mathrm{~N}$, for the first time detected in a solartype protostar. It is indeed only ten times lower than $\mathrm{HC}_{3} \mathrm{~N}$ in the inner warm region and similar to the $\mathrm{HC}_{3} \mathrm{~N}$ abundance in the outer cold envelope. When compared to other protostellar sources where $\mathrm{HC}_{5} \mathrm{~N}$ has been detected, this ratio is not so anomalous. Figure 8 shows the two abundances in several protostellar sources and cold clouds. The two coldest sources have both an $\mathrm{HC}_{5} \mathrm{~N} / \mathrm{HC}_{3} \mathrm{~N} \sim 1$, while in the other sources this ratio is $\sim 0.1$. However, our chemical model fails to reproduce such a high abundance of $\mathrm{HC}_{5} \mathrm{~N}$, even though we included part of the most updated chemical network, recently revised by Loison et al. (2014). Evidently, we are missing key reactions that form this species.

\section{3. $\mathrm{HC}_{3} \mathrm{~N}$ deuteration}

Finally, we detected for the first time the $\mathrm{DC}_{3} \mathrm{~N}$ in a solar-type protostar. The deuteration of $\mathrm{HC}_{3} \mathrm{~N}$ is about $50 \%$ in the outer cold envelope and lower than $5 \%$ in the warm part. This also provides us with important clues on the present and past history of IRAS 16293. First, the high deuteration in the cold envelope tells us that this is a present-day product, namely it is caused by a cold and CO-depleted gas, in agreement with previous observations and theoretical predictions (e.g. Ceccarelli et al. 2014a).

The very low deuteration in the warm part is, in contrast, more intriguing. It cannot be the result of an initial high deuteration that has been diminished by gas-phase reactions, because, as we have argued above, $\mathrm{HC}_{3} \mathrm{~N}$ is the result of the volcano sublimation, which occurred because of a quick, $\leq 10^{3} \mathrm{yr}$, heating of the dust. This means that it must be a fossil from the dust before the warming phase. This is probably one of the few very clear cases of fossil deuteration where there are no doubts that it is pristine (another one, to our knowledge, is the one of HDCO in the protostellar molecular shock L1157-B1; Fontani et al. 2014). The deuteration in IRAS 16293 is very high in general, with a doubly deuterated ratio of formaldehyde of $\sim 30 \%$, for example (Ceccarelli et al. 1998), and a triply deuterated ratio of methanol of a few percent (Parise et al. 2004). While at present we still do not have a clear measure of the formaldehyde deuteration in the warmer and colder envelope separately (e.g. Ceccarelli et al. 2001), methanol should be entirely concentrated in the warm region. However, recently, deuterated formamide $\left(\mathrm{NH}_{2} \mathrm{CDO}\right.$ and NHDCHO) has been detected by ALMA in IRAS 16293, and the observations clearly show that formamide line emission is associated with the warm hot corino (Coutens et al. 2016). The measured deuteration ratio is a few percent, similar to that found in $\mathrm{HC}_{3} \mathrm{~N}$ in the present work. Therefore, the molecular deuteration is a complex phenomenon even within the same source.

If our reasoning above is correct, namely the present-day gaseous $\mathrm{HC}_{3} \mathrm{~N}$ is the sublimation product of previously frozen $\mathrm{HC}_{3} \mathrm{~N}$, then its relatively low deuteration tells us that this species is an early chemical product, namely it was formed in a time when the gas temperature was not too low and, mostly importantly, the $\mathrm{CO}$ was not depleted yet (see e.g. Ceccarelli et al. 2014a). This is in perfect agreement with the models of $\mathrm{HC}_{3} \mathrm{~N}$ formation, which show that $\mathrm{HC}_{3} \mathrm{~N}$ is abundantly formed before the full trapping of carbon into $\mathrm{CO}$ (see e.g. Loison et al. 2014, and Sect. 5). Therefore, the low $\mathrm{DC}_{3} \mathrm{~N} / \mathrm{HC}_{3} \mathrm{~N}$ in the warm part is well explained by the picture that abundant $\mathrm{HC}_{3} \mathrm{~N}$ has been formed during the tenuous molecular cloud phase and then frozen onto the ice mantles when the condensation that gave birth to IRAS 16293 increased in density while it decreased in temperature. Then, we predict that the deuteration of $\mathrm{HC}_{3} \mathrm{~N}$ in the hot corino regions could be a good probe of the timescale of the collapse as well. Of course, detailed modelling will be necessary to quantify this prediction.

Meanwhile, the collection of previous measures of $\mathrm{DC}_{3} \mathrm{~N} / \mathrm{HC}_{3} \mathrm{~N}$, shown in Fig. 9, provides support to our thesis. With the exception of the cold envelope of IRAS 16293, all other sources where $\mathrm{DC}_{3} \mathrm{~N}$ has been observed, possess a $\mathrm{HC}_{3} \mathrm{~N}$ deuteration of a few percent, in agreement with the hypothesis that $\mathrm{HC}_{3} \mathrm{~N}$ is an early chemical product.

\section{Conclusions}

We detected several lines from cyanoacetylene $\left(\mathrm{HC}_{3} \mathrm{~N}\right)$ and cyanodiacetylene $\left(\mathrm{HC}_{5} \mathrm{~N}\right)$, and provided an upper limit to the abundance of cyanotriacetylene $\left(\mathrm{HC}_{7} \mathrm{~N}\right)$ and other undetected 


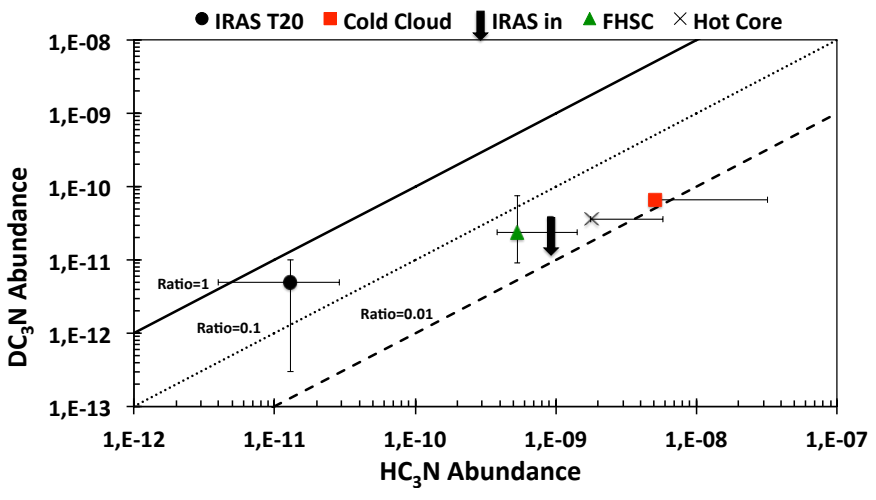

Fig. 9. Abundance of $\mathrm{DC}_{3} \mathrm{~N}$ as a function of the abundance of $\mathrm{HC}_{3} \mathrm{~N}$ in different protostellar and cold sources. The symbols are the same as those in Fig. 8.

cyanopolyynes. We also reported the first detection of deuterated cyanoacetylene, $\mathrm{DC}_{3} \mathrm{~N}$, in a solar-type protostar. In contrast, we did not detect any ${ }^{13} \mathrm{C}$ cyanopolyyne isotopologue. We found that the $\mathrm{HC}_{3} \mathrm{~N}$ abundance is roughly constant $\left(\sim 1.3 \times 10^{-11}\right)$ in the outer cold envelope of IRAS 16293-2422 and it increases, as a step-function, by about a factor 100 in the inner region where the dust temperature exceeds $80 \mathrm{~K}$. The $\mathrm{HC}_{5} \mathrm{~N}$ has an abundance similar to $\mathrm{HC}_{3} \mathrm{~N}$ in the outer envelope and about a factor of ten lower in the inner region.

A comparison with a chemical model provided constraints on the oxygen and carbon gaseous abundance in the outer envelope and, most importantly, on the age of the source. The $\mathrm{HC}_{3} \mathrm{~N}$ abundance derived in the inner region and where the jump occurs also provided strong constraints on the time taken for the dust to warm up to $80 \mathrm{~K}$, which has to be less than $\sim 10^{3}-10^{4} \mathrm{yr}$.

Finally, the cyanoacetylene deuteration is about $50 \%$ in the outer envelope and $\sim 5 \%$ in the warm inner region. The relatively low deuteration in the warm region suggests that we are seeing an almost pristine fossil of the $\mathrm{HC}_{3} \mathrm{~N}$, abundantly formed in the tenuous phase of the pre-collapse and then frozen into the grain mantles at a later phase.

Acknowledgements. This research was supported in part by the National Science Foundation under Grant No. NSF PHY11-25915. We acknowledge the financial support from the university of Al-Muthana and ministry of higher education and scientific research in Iraq. E.M. acknowledges support from the Brazilian agency FAPESP under grants 2014/22095-6 and 2015/22254-0.

\section{References}

Aladro, R., Martín-Pintado, J., Martín, S., Mauersberger, R., \& Bayet, E. 2011, A\&A, 525, A89

Asplund, M., Grevesse, N., Sauval, A. J., \& Scott, P. 2009, ARA\&A, 47, 481

Bachiller, R., \& Pérez Gutiérrez, M. 1997, ApJ, 487, L93

Bell, M. B., Feldman, P. A., Travers, M. J., et al. 1997, ApJ, 483, L61

Ben Abdallah, D., Najar, F., Jaidane, N., Dumouchel, F., \& Lique, F. 2012, MNRAS, 419, 2441

Bockelée-Morvan, D., Lis, D. C., Wink, J. E., et al. 2000, A\&A, 353, 1101

Brack, A. 1998, in The Molecular Origins of Life: Assembling Pieces of the Puzzle (Cambridge University Press)
Caselli, P., \& Ceccarelli, C. 2012, A\&ARv, 20, 56

Caselli, P., Walmsley, C. M., Terzieva, R., \& Herbst, E. 1998, ApJ, 499, 234

Caux, E., Kahane, C., Castets, A., et al. 2011, A\&A, 532, A23

Cazaux, S., Tielens, A. G. G. M., Ceccarelli, C., et al. 2003, ApJ, 593, L51

Ceccarelli, C., Hollenbach, D. J., \& Tielens, A. G. G. M. 1996, ApJ, 471, 400

Ceccarelli, C., Castets, A., Loinard, L., Caux, E., \& Tielens, A. G. G. M. 1998, A\&A, 338, L43

Ceccarelli, C., Castets, A., Caux, E., et al. 2000, A\&A, 355, 1129

Ceccarelli, C., Loinard, L., Castets, A., et al. 2001, A\&A, 372, 998

Ceccarelli, C., Maret, S., Tielens, A. G. G. M., Castets, A., \& Caux, E. 2003, A\&A, 410, 587

Ceccarelli, C., Caselli, P., Bockelée-Morvan, D., et al. 2014a, Protostars and Planets VI, 859

Ceccarelli, C., Dominik, C., López-Sepulcre, A., et al. 2014b, ApJ, 790, L1 Chandler, C. J., Brogan, C. L., Shirley, Y. L., \& Loinard, L. 2005, ApJ, 632, 371 Clarke, D. W., \& Ferris, J. P. 1995, Icarus, 115, 119

Collings, M. P., Anderson, M. A., Chen, R., et al. 2004, MNRAS, 354, 1133 Cordiner, M. A., Charnley, S. B., Wirström, E. S., \& Smith, R. G. 2012, ApJ, 744,131

Coutens, A., Jørgensen, J. K., van der Wiel, M. H. D., et al. 2016, A\&A, 590, L6

Crimier, N., Ceccarelli, C., Maret, S., et al. 2010, A\&A, 519, A65

Doty, S. D., Schöier, F. L., \& van Dishoeck, E. F. 2004, A\&A, 418, 1021

Esplugues, G. B., Cernicharo, J., Viti, S., et al. 2013, A\&A, 559, A51

Faure, A., Lique, F., \& Wiesenfeld, L. 2016, MNRAS, 460, 2103

Fontani, F., Codella, C., Ceccarelli, C., et al. 2014, ApJ, 788, L43

Friesen, R. K., Medeiros, L., Schnee, S., et al. 2013, MNRAS, 436, 1513

García-Rojas, J., \& Esteban, C. 2007, ApJ, 670, 457

Goesmann, F., Rosenbauer, H., Bredehöft, J. H., et al. 2015, Science, 349, 6247

Jaber, A. A., Ceccarelli, C., Kahane, C., \& Caux, E. 2014, ApJ, 791, 29

Jørgensen, J. K., Schöier, F. L., \& van Dishoeck, E. F. 2004, A\&A, 416, 603

Jørgensen, J. K., Bourke, T. L., Nguyen Luong, Q., \& Takakuwa, S. 2011, A\&A, 534, A 100

Loinard, L., Torres, R. M., Mioduszewski, A. J., \& Rodríguez, L. F. 2008, ApJ, 675, L29

Loinard, L., Zapata, L. A., Rodríguez, L. F., et al. 2013, MNRAS, 430, L10

Loison, J.-C., Wakelam, V., Hickson, K. M., Bergeat, A., \& Mereau, R. 2014, MNRAS, 437, 930

Loomis, R. A., Shingledecker, C. N., Langston, G., et al. 2016, MNRAS, 463, 4175

Lunine. 2009, EPJ Web Conf., 1, 267

Marr, J. M., Wright, M. C. H., \& Backer, D. C. 1993, ApJ, 411, 667

McElroy, D., Walsh, C., Markwick, A. J., et al. 2013, A\&A, 550, A36

Miettinen, O. 2014, A\&A, 562, A3

Mizuno, A., Fukui, Y., Iwata, T., Nozawa, S., \& Takano, T. 1990, ApJ, 356, 184

Müller, H. S. P., Schlöder, F., Stutzki, J., \& Winnewisser, G. 2005, J. Mol. Struct., 742,215

Mundy, L. G., Wootten, A., Wilking, B. A., Blake, G. A., \& Sargent, A. I. 1992, ApJ, 385, 306

Parise, B., Castets, A., Herbst, E., et al. 2004, A\&A, 416, 159

Pickett, H. M., Poynter, R. L., Cohen, E. A., et al. 1998, J. Quant. Spectr. Rad. Transf., 60, 883

Pineda, J. E., Maury, A. J., Fuller, G. A., et al. 2012, A\&A, 544, L7

Sakai, N., Sakai, T., Hirota, T., \& Yamamoto, S. 2008, ApJ, 672, 371

Schöier, F. L., Jørgensen, J. K., van Dishoeck, E. F., \& Blake, G. A. 2002, A\&A, 390, 1001

Snell, R. L., Schloerb, F. P., Young, J. S., Hjalmarson, A., \& Friberg, P. 1981, ApJ, 244, 45

Suzuki, H., Yamamoto, S., Ohishi, M., et al. 1992, ApJ, 392, 551

van Dishoeck, E. F., Blake, G. A., Draine, B. T., \& Lunine, J. I. 1993, in Protostars and Planets III, eds. E. H. Levy, \& J. I. Lunine, 163

Viti, S., Collings, M. P., Dever, J. W., McCoustra, M. R. S., \& Williams, D. A. 2004, MNRAS, 354, 1141

Wakelam, V., Loison, J.-C., Herbst, E., et al. 2015, ApJS, 217, 20

Wernli, M., Wiesenfeld, L., Faure, A., \& Valiron, P. 2007, A\&A, 464, 1147

Winstanley, N., \& Nejad, L. A. M. 1996, Ap\&SS, 240, 13

Wootten, A. 1989, ApJ, 337, 858

Zapata, L. A., Loinard, L., Rodríguez, L. F., et al. 2013, ApJ, 764, L14 


\section{Appendix A: Figures that show the $\mathrm{HC}_{3} \mathrm{~N}$ modelling}

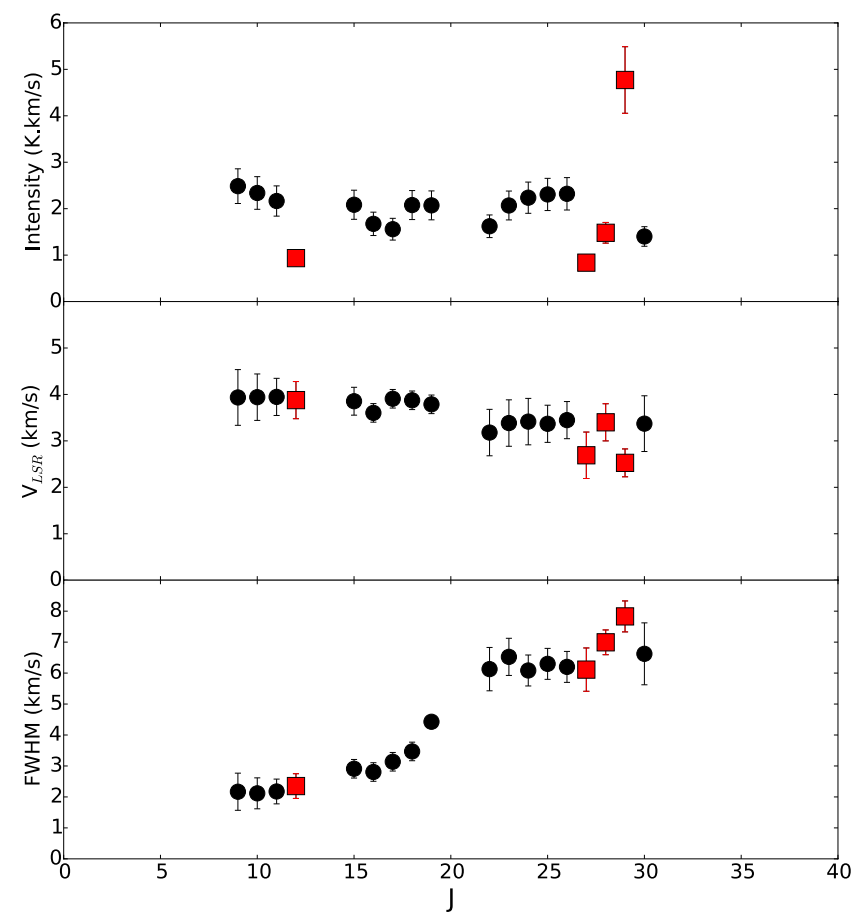

Fig. A.1. $\mathrm{HC}_{3} \mathrm{~N}$ line intensity (upper panel), rest velocity $V_{\mathrm{LSR}}$ (middle panel), and FWHM (bottom panel) as a function of the upper $\mathrm{J}$ of the transition. The red squares show the lines that have been discarded because they did not satisfy all criteria 3 to 5 of Sect. 3.2 (see text).
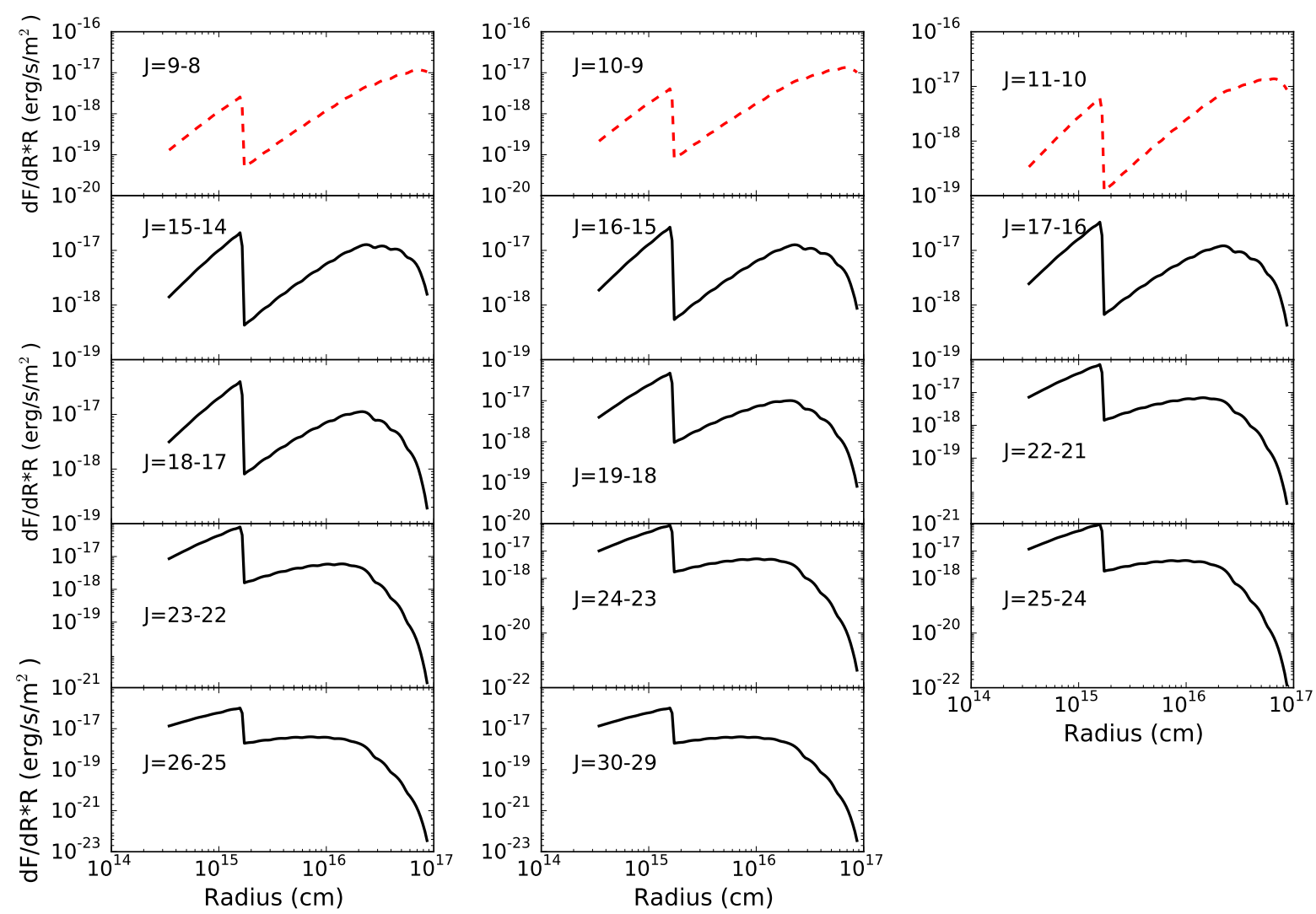

Fig. A.2. Predicted contribution to the integrated line intensity $(\mathrm{d} F / \mathrm{d} r * r)$ of a shell at a radius $r$ for the $\mathrm{HC}_{3} \mathrm{~N}$ lines. This model corresponds to $\alpha=0, T_{\text {jump }}=80 \mathrm{~K}, X_{\text {in }}=3.6 \times 10^{-10}$, and $X_{\text {out }}=6.0 \times 10^{-11}$. The three upper red dashed curves show an increasing emission towards the maximum radius and very likely contaminated by the molecular cloud (see Sect. 4.2.1). 
A. Jaber Al-Edhari et al.: History of the solar-type protostar IRAS 16293-2422 as told by the cyanopolyynes
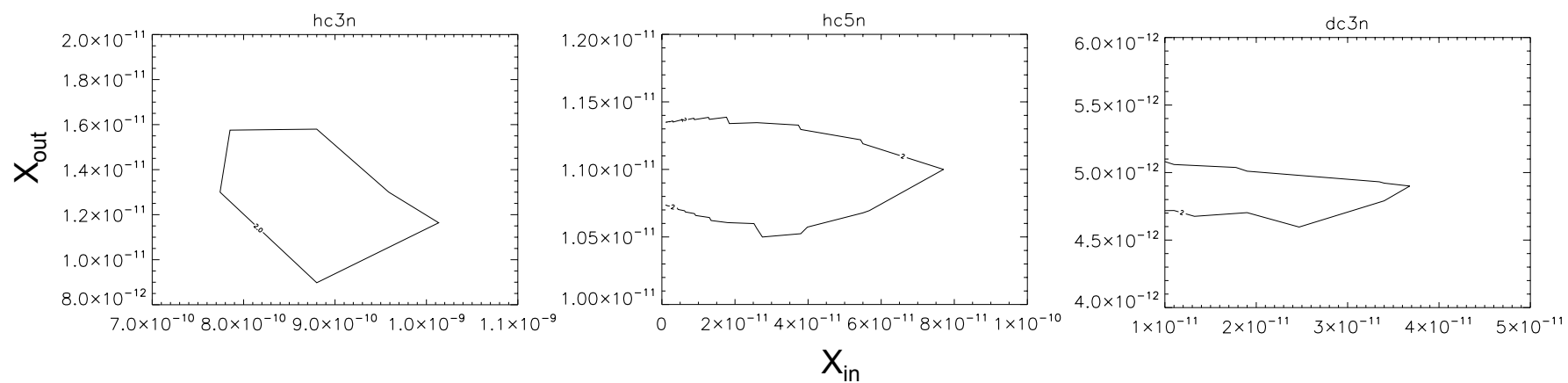

Fig. A.3. $\chi^{2}$ contour plots for $\mathrm{HC}_{3} \mathrm{~N}$ (left), $\mathrm{HC}_{5} \mathrm{~N}$ (middle) and $\mathrm{DC}_{3} \mathrm{~N}$ (right) as a function of $X_{\text {in }}$ and $X_{\text {out }}$. The predictions refer to a model with $T_{\text {jump }}=80 \mathrm{~K}$ and $\alpha=0$.

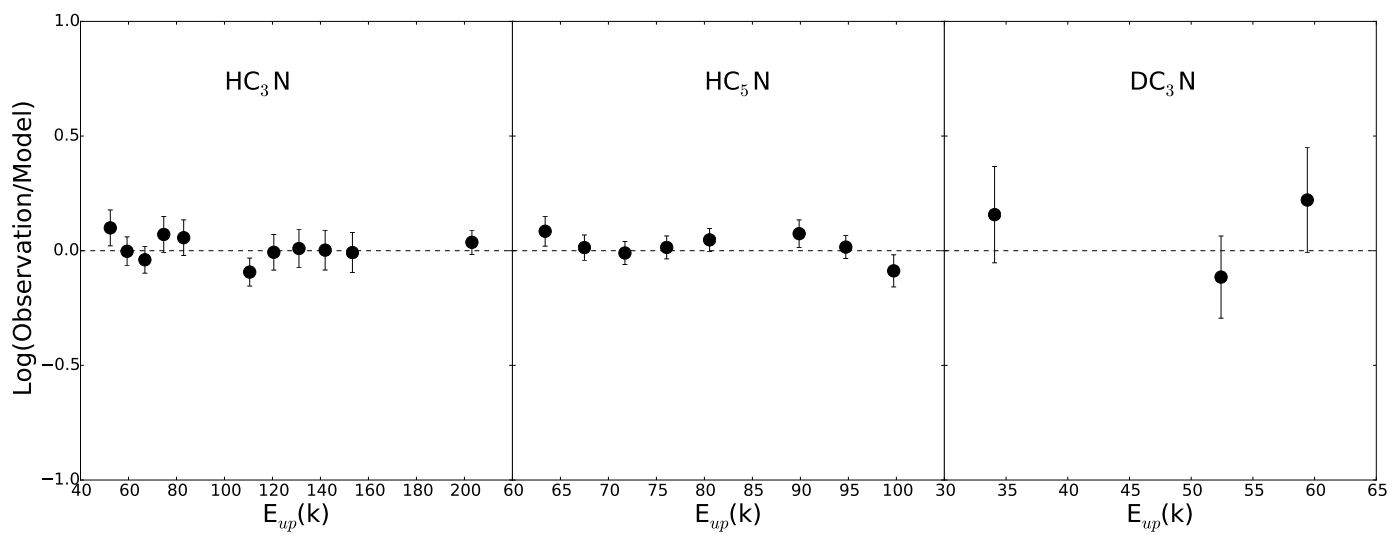

Fig. A.4. Ratio of the observed over predicted line flux as a function of the upper level energy of the transition for the model $2\left(T_{\text {jump }}=80 \mathrm{~K}\right.$ and $\alpha=0$ ), for $\mathrm{HC}_{3} \mathrm{~N}(l e f t), \mathrm{HC}_{5} \mathrm{~N}$ (middle), and $\mathrm{DC}_{3} \mathrm{~N}$ (right), respectively.
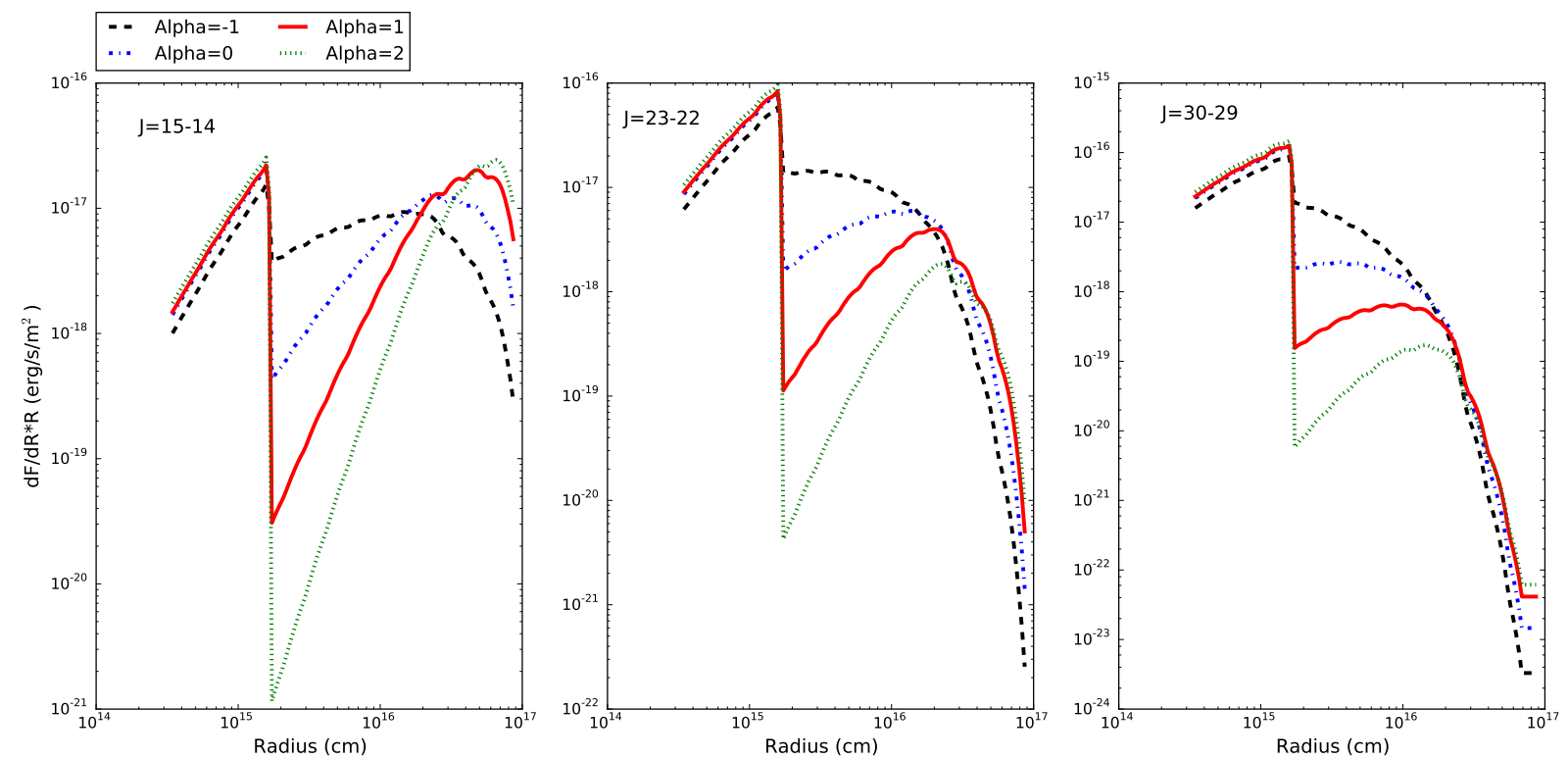

Fig. A.5. Velocity-integrated flux emitted from each shell at a radius $r(\mathrm{~d} F / \mathrm{d} r * r)$ as a function of the radius for the $\mathrm{HC}_{3} \mathrm{~N}$ four best NLTE model, for three low, middle, and high values of $J$. 